\title{
Population Coding of Natural Electrosensory Stimuli by Midbrain Neurons
}

\author{
${ }^{(}$Michael G. Metzen and ${ }^{\circledR}$ Maurice J. Chacron \\ Department of Physiology, McGill University, Montreal, Quebec H3G 1Y6, Canada
}

Natural stimuli display spatiotemporal characteristics that typically vary over orders of magnitude, and their encoding by sensory neurons remains poorly understood. We investigated population coding of highly heterogeneous natural electrocommunication stimuli in Apteronotus leptorhynchus of either sex. Neuronal activities were positively correlated with one another in the absence of stimulation, and correlation magnitude decayed with increasing distance between recording sites. Under stimulation, we found that correlations between trial-averaged neuronal responses (i.e., signal correlations) were positive and higher in magnitude for neurons located close to another, but that correlations between the trial-to-trial variability (i.e., noise correlations) were independent of physical distance. Overall, signal and noise correlations were independent of stimulus waveform as well as of one another. To investigate how neuronal populations encoded natural electrocommunication stimuli, we considered a nonlinear decoder for which the activities were combined. Decoding performance was best for a timescale of $6 \mathrm{~ms}$, indicating that midbrain neurons transmit information via precise spike timing. A simple summation of neuronal activities (equally weighted sum) revealed that noise correlations limited decoding performance by introducing redundancy. Using an evolution algorithm to optimize performance when considering instead unequally weighted sums of neuronal activities revealed much greater performance values, indicating that midbrain neuron populations transmit information that reliably enable discrimination between different stimulus waveforms. Interestingly, we found that different weight combinations gave rise to similar discriminability, suggesting robustness. Our results have important implications for understanding how natural stimuli are integrated by downstream brain areas to give rise to behavioral responses.

Key words: correlated variability; correlations; natural stimuli; neural coding; population coding; weakly electric fish

Significance Statement

We show that midbrain electrosensory neurons display correlations between their activities and that these can significantly impact performance of decoders. While noise correlations limited discrimination performance by introducing redundancy, considering unequally weighted sums of neuronal activities gave rise to much improved performance and mitigated the deleterious effects of noise correlations. Further analysis revealed that increased discriminability was achieved by making trialaveraged responses more separable, as well as by reducing trial-to-trial variability by eliminating noise correlations. We further found that multiple combinations of weights could give rise to similar discrimination performances, which suggests that such combinatorial codes could be achieved in the brain. We conclude that the activities of midbrain neuronal populations can be used to reliably discriminate between highly heterogeneous stimulus waveforms.

\section{Introduction}

Understanding how neural populations process incoming sensory information remains a central problem in systems neuroscience. Indeed, population coding has been studied extensively

Received Aug. 25, 2020; revised Feb. 27, 2021; accepted Mar. 1, 2021.

Author contributions: M.G.M. and M.J.C. designed research; M.G.M. performed research; M.J.C. contributed unpublished reagents/analytic tools; M.G.M. analyzed data; M.G.M. and M.J.C. wrote the paper.

This research was supported by Canadian Institutes of Health Research Project Grant 159694 (M.G.M., M.J.C.).

The authors declare no competing financial interests.

Correspondence should be addressed to Maurice J. Chacron at maurice.chacron@mcgill.ca.

https://doi.org/10.1523/JNEUROSCI.2232-20.2021

Copyright $\odot 2021$ the authors both theoretically and experimentally (Georgopoulos et al., 1986; Abbott and Dayan, 1999; Averbeck et al., 2006; Ecker et al., 2010; Kohn et al., 2016; Mendels and Shamir, 2018). Of particular interest are the effects of correlations between the trial-to-trial variabilities of neural responses to repeated presentations of a given stimulus (i.e., "noise correlations"). On the one hand, noise correlations are thought to introduce redundancy between neural activities and thus should be eliminated or, at the very least, reduced in magnitude (Averbeck and Lee, 2006; Ecker et al., 2010). However, noise correlations can vary depending on stimulus attributes, which can introduce synergy to benefit coding (Moreno-Bote et al., 2014; Lin et al., 2015; Franke et al., 2016; Zylberberg et al., 2016). However, most studies of population coding to date have considered artificial stimuli because of their 
relative simplicity. As such, population coding of natural stimuli that are often complex because of their rich spatiotemporal structure (Attias and Schreiner, 1997; Simoncelli and Olshausen, 2001; Mante et al., 2005; Fotowat et al., 2013; Henninger et al., 2018) has been much less investigated to date (Mizrahi et al., 2014; Yoshida and Ohki, 2020). In particular, because singleneuron responses to natural stimuli cannot be predicted from their responses to artificial stimuli in general (Mizrahi et al., 2014), there is reason to believe that the strategies used by neural populations to encode natural stimuli will differ from those used to encode artificial stimuli.

Gymnotiform wave-type weakly electric fish generate an electric field around their body through an electric organ discharge (EOD) and can sense perturbations of this field through an array of electroreceptors distributed on their skin. Electroreceptors make synaptic contact onto pyramidal cells within the electrosensory lateral line lobe (ELL), which in turn project to downstream brain areas such as the torus semicircularis (TS; Bell and Maler, 2005). There are $~ 50$ different neuron types within TS described in the literature that contribute to electrosensory processing (Carr et al., 1981; Carr and Maler, 1985), which translates to large heterogeneities in responses to sensory input (Vonderschen and Chacron, 2011). Natural electrosensory stimuli comprise those caused by objects such as prey (Nelson and MacIver, 1999) as well as those caused by conspecifics (Zakon et al., 2002; for review, see Metzen, 2019). In the latter case, natural electrocommunication stimuli (i.e., "chirps") consist of transient increases in EOD frequency that occur on top of the underlying sinusoidal background beat (Zupanc and Maler, 1993; Engler et al., 2000; Bastian et al., 2001; for review, see Zupanc, 2002; Kolodziejski et al., 2005). Chirp attributes (e.g., amplitude, duration) vary over a wide range for a given species (Zupanc and Maler, 1993; Zakon et al., 2002), which gives rise to a rich repertoire of different stimulus waveforms (for review, see Metzen, 2019). The coding of chip stimuli by electrosensory neurons has been the focus of previous studies (Benda et al., 2005, 2006; Marsat et al., 2009; Marsat and Maler, 2010; Vonderschen and Chacron, 2011; Walz et al., 2013, 2014; Metzen et al., 2016, 2020; Metzen and Chacron, 2017 for review, see Metzen, 2019). However, these have either considered single neurons or used nonsimultaneous recordings to study population coding, such that the effects of noise correlations were ignored.

\section{Materials and Methods}

Ethics statement. All animal procedures were approved by the McGill University animal care committee (\#5285) and were performed in accordance with the guidelines of the Canadian Council on Animal Care. All fish were immobilized by an intramuscular injection of $0.1-$ $0.5 \mathrm{mg}$ of tubocurarine before experiments.

Animals. The wave-type weakly electric fish Apteronotus leptorhynchus $(N=3)$ of either sex was used exclusively in this study. Animals of either sex were purchased from tropical fish suppliers and were housed in groups $(2-10)$ at controlled water temperatures $\left(26-29^{\circ} \mathrm{C}\right)$ and conductivities (300-800 $\mu \mathrm{S} / \mathrm{cm})$ according to published guidelines (Hitschfeld et al., 2009).

Surgery. Surgical procedures have been described in detail previously (Chacron et al., 2003; Toporikova and Chacron, 2009; Metzen et al., 2015). Briefly, 0.1-0.5 mg of tubocurarine (Sigma-Aldrich) was injected intramuscularly to immobilize the animals for electrophysiology experiments. The animals were then transferred to an experimental tank $(30 \times$ $30 \times 10 \mathrm{~cm}$ ) containing water from the animal's home tank and respired by a constant flow of oxygenated water through their mouth at a flow rate of $10 \mathrm{ml} / \mathrm{min}$. Subsequently, the head of the animal was locally anesthetized with lidocaine ointment (5\%; AstraZeneca), the skull was partly exposed, and a small window $\left(\sim 5 \mathrm{~mm}^{2}\right)$ was opened over the midbrain.

Stimulation. The electric organ discharge of A. leptorhynchus is neurogenic, and therefore is not affected by the injection of curare. All stimuli consisted of amplitude modulations (AMs; or beats) of the EOD of the animal and were produced by triggering a function generator to emit one cycle of a sine wave for each zero crossing of the EOD, as done previously (Bastian et al., 2002). The frequency of the emitted sine wave was set slightly higher $(30 \mathrm{~Hz})$ than that of the EOD, which allowed the output of the function generator to be synchronized to the discharge of the animal. The emitted sine wave was subsequently multiplied with the desired AM waveform (MT3 Multiplier, Tucker-Davis Technologies), and the resulting signal was isolated from the ground (A395 Linear Stimulus Isolator, World Precision Instruments). The isolated signal was then delivered through a pair of chloridized silver wire electrodes placed $15 \mathrm{~cm}$ away from the animal on either side of the recording tank perpendicular to the rostrocaudal axis of the fish.

To elicit neuronal responses, we generated chirps with different attributes by systematically varying the chirp duration $(8,11,14,17,20$, $53,141,376$, and $1000 \mathrm{~ms})$, the amplitude $(10,35,60,85$, and $110 \mathrm{~Hz})$, and the phase $\left(0^{\circ}, 45^{\circ}, 90^{\circ}, 135^{\circ}, 180^{\circ}, 225^{\circ}, 270^{\circ}\right.$, and $\left.315^{\circ}\right)$ of the underlying beat cycle at which the chirp occurs (Fig. 1b). Importantly, when varying chirp duration or amplitude, we considered chirps occurring at either phase $90^{\circ}$ or $270^{\circ}$ of the beat cycle, on top of a sinusoidal beat with frequency $f_{\text {beat }}=4 \mathrm{~Hz}$, as done previously (Vonderschen and Chacron, 2011; Metzen et al., 2016). As such, we used a total of 36 different chirp waveforms (18 for chirp duration, 10 for chirp amplitude, 8 for chirp phase). Parameter ranges were chosen to contain those observed in previous studies (Zupanc and Maler, 1993; Engler and Zupanc, 2001; Zupanc et al., 2006; Metzen et al., 2020), including durations that cover those of other electrocommunication signals such as long frequency rises (Kolodziejski et al., 2005) or long chirps (Henninger et al., 2018). It is important to note that the chirp amplitude is not equivalent to the actual spectral frequency content of the resulting AM stimulus, which is 50$100 \mathrm{~Hz}$ (Zupanc and Maler, 1993). We chose a $4 \mathrm{~Hz}$ beat because, as mentioned above, this was the frequency used in previous studies (Metzen et al., 2016, 2020). We note that the chirp stimuli considered here were consistently most frequently produced when the frequency difference between both animals was lowest for various conditions (Zupanc and Maler, 1993; Bastian et al., 2001; Engler and Zupanc, 2001; Hupé and Lewis, 2008). Previous studies have furthermore demonstrated a sex dimorphism in EOD frequency for A. leptorhynchus in that males have significantly higher EOD frequencies than females (Meyer et al., 1987; Zupanc et al., 2014). As such, same-sex encounters will on average lead to lower beat frequencies than opposite-sex encounters (Benda et al., 2006). To measure the stimulus intensity, a small dipole was placed close to the skin of the animal. Stimulus intensity was adjusted to produce changes in EOD amplitude that were $\sim 20 \%$ of the baseline level, as done previously (Metzen et al., 2016, 2020; Metzen and Chacron, 2017). Finally, each chirp stimulus waveform (i.e., a chirp with given duration, amplitude, or phase) was presented 60 times (i.e., 60 trials) with $\sim 1.5 \mathrm{~s}$ between subsequent presentations, followed by another waveform. We note that midbrain neurons do not display adaptation to repeated presentations of a given chirp stimulus (Vonderschen and Chacron, 2011; Metzen et al., 2016; see also Fig. 4).

Recordings. Simultaneous extracellular recordings from TS neurons were made using Neuropixel probes (Imec). Recordings were digitized at $30 \mathrm{kHz}$ using spikeGLX (Janelia Research Campus, Howard Hughes Medical Institute) and stored on a hard drive for further analysis. Spikes were sorted using Spike2 (Cambridge Electronic Design), because we did not observe any drifting of spiking activity across channels or record more than a maximum of three neurons on any single channel. In fact, most channels contained a single well isolated neuron (Fig. $2 a$, right). Overall, we recorded from a total of $N=158$ neurons (40 for session 1, 28 chirp stimuli; 77 for session 2, 36 chirp stimuli; and 41 for session 3, 36 chirp stimuli). However, not all neurons were stable over the entire stimulus protocol such that only a subpopulation could be extracted that received a common set of stimuli ( 28 for session $1 ; 27$ for session $2 ; 2$ for 
a

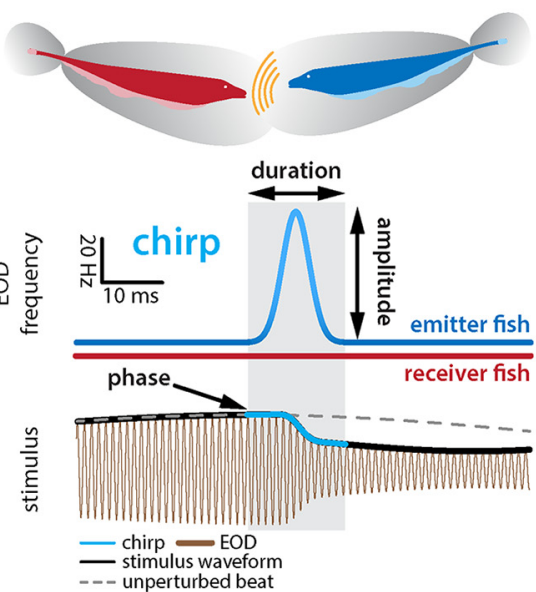

b
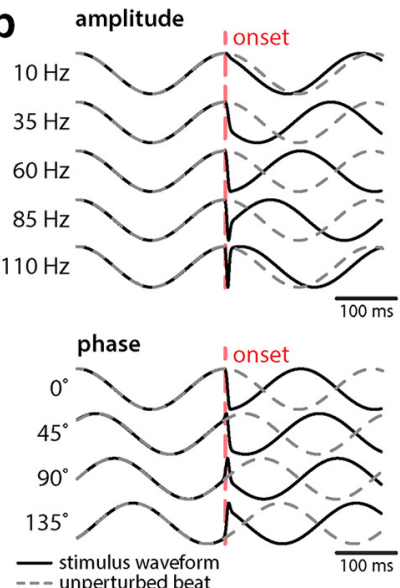

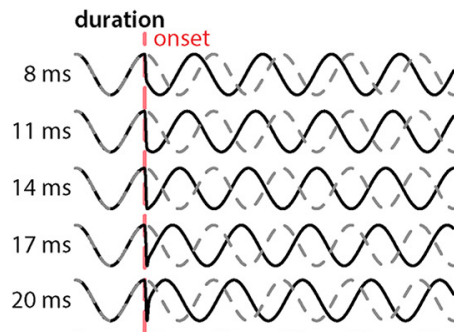

$53 \mathrm{~ms}$ 甪分 $141 \mathrm{~ms}$ Mind

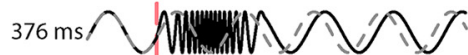

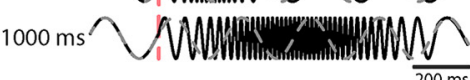

Figure 1. Chirp-induced AM waveforms are highly heterogeneous. $\boldsymbol{a}$, During a chirp event, the EOD frequency (middle, blue trace) of the emitter fish (top, blue) is transiently increased by the maximum of amplitude for a brief duration (light blue, shaded box) while the EOD frequency (middle, red trace) of the receiver fish (top, red) remains constant. This can be characterized by the duration and amplitude of the frequency excursion. The chirp results in a phase advance of the beat (i.e., the beat will reach its maximum value earlier than if no chirp occurred; compare solid black with dashed gray). The chirp is shown in light blue. $\boldsymbol{b}$, AM waveforms obtained by varying different parameters (black curves; left top, fixed chirp duration and phase, varying chirp amplitude; left bottom, fixed chirp duration and amplitude, varying chirp phase; right, fixed chirp phase and amplitude, varying chirp duration). Also shown are unperturbed beat waveforms (dashed gray). Note that different chirps give rise to different phase advances of the beat. The red dashed horizontal lines indicate chirp onset.

session 3). The average baseline firing rate for our dataset was $14.0 \pm$ 16.8 spikes (spk)/s, which is slightly higher than those obtained in previous studies (Vonderschen and Chacron, 2011; McGillivray et al., 2012; Metzen et al., 2016), which is in part because of the fact that we did not exclude neurons that did not respond to the stimuli presented. Overall, similar results were seen for all three recording sessions. Distances between neurons were computed as the distance between probe sites on which the action potentials of recorded neurons were highest. We note that the geometry of the recording channels on the Neuropixel probes could introduce "gaps" when calculating neuronal distances, as not all channels may contain sortable spikes. Based on the fact that the Neuropixel probe consists of 384 parallel recording channels (checkerboard, two columns) on a 10-mm-long shank with distances of $16 \mu \mathrm{m}$ (column) and $20 \mu \mathrm{m}$ (row; Jun et al., 2017), distances primarily reflect vertical distances along the probe shaft. The probe was angled at $\sim 15^{\circ}$ with respect to vertical in order for the shaft to be approximately perpendicular to the layers of TS and advanced between 1500 and $2000 \mu \mathrm{m}$ into TS with reference to the probe tip. As such, the reported distances primarily reflect distances across rather than within TS layers. Neurons were recorded at depths between 300 and $1900 \mu \mathrm{m}$ from the brain surface. Of all neurons recorded from, $\sim 60 \%$ were located within the first $1000 \mu \mathrm{m}$ and were thus mostly located within the first five layers of TS.

Data analysis. Sorted and curated spike times for each neuron were converted into "binary" sequences sampled at $2 \mathrm{kHz}$. The content of a given bin was set to 2000 if a spike occurred within it and 0 otherwise. We note that, as the bin width was smaller than the refractory period of TS neurons, there can be at most one spike occurring during any given bin. The time-dependent firing rate of neuron $i, X_{i}(t)$, was obtained by filtering the binary sequence using a box-car filter with $6 \mathrm{~ms}$. Latencies for single-neuron responses were estimated from the first spike after stimulus onset.

Determining neuron response type. To quantify the response to the chirp, we used the chirp selectivity index (CSI) to determine whether a given neuron responded to the chirp as done previously (Vonderschen and Chacron, 2011), as follows:

$$
\mathrm{CSI}=\frac{R_{\text {chirp }}-R_{\text {beat }}}{R_{\text {chirp }}+R_{\text {beat }}}
$$

where $R_{\text {chirp }}$ is the maximum firing rate obtained in a peristimulus time histogram (PSTH) during a time window of $100 \mathrm{~ms}$ starting with chirp onset, and $R_{\text {beat }}$ is the maximum firing rate obtained during the ongoing beat in a time window of $2 \mathrm{~s}$ ending on chirp onset. The CSI ranges between -1 and 1 , representing perfect selectivity for the beat at -1 and the chirp at 1 . To measure the selectivity of a neuron to multiple chirp stimuli, the average CSI was used, as follows:

$$
\mathrm{CSI}_{\mathrm{avg}}=\frac{1}{N} \sum_{i=1}^{N} \operatorname{CSI}_{i}
$$

where $N$ is the number of chirp stimuli tested and $\mathrm{CSI}_{i}$ is the CSI to chirp stimulus $i$.

We also quantified neuronal gain to the $4 \mathrm{~Hz}$ background beat, which was measured as the ratio between the amplitude of the sinusoidal beat stimulus as extracted from the dipole recording by performing a Hilbert transform and low-pass filtering and the amplitude of the neuronal firing rate modulation.

CSI and gain were used to capture the response types of all neurons recorded. Because a negative CSI value only indicates the preference of a neuron response to the beat but not to the chirp, it is thus not able to correctly differentiate between nonresponding and beat-responding neurons. We thus used the gain to the beat to further distinguish between these two response types. As such, neurons that had an average CSI of $\geq 0.1$ for all chirp stimuli presented where regarded as responding to the chirp, as done previously (Vonderschen and Chacron, 2011). Neurons with gain to the $4 \mathrm{~Hz}$ background beat $\geq 2 \mathrm{spk} / \mathrm{s}(\mathrm{mV} / \mathrm{cm})$ where regarded as responding to the beat. Based on their response characteristics to the beat and the chirp, neurons were classified as follows: (1) beat responders ["br"; neurons that exclusively responded to the beat, but not to the chirp (i.e., CSI $<0.1$ and gain $\geq 2 \mathrm{spk} / \mathrm{s}(\mathrm{mV} / \mathrm{cm}) ; 16(57 \%)$ for session 1; 8 for session 2 (30\%); 2 for session 3 (100\%)]; (2) beat and chirp responders ["bcr"; neurons that responded to the beat as well as to the chirp (i.e., CSI $\geq 0.1$ and gain $\geq 2 \mathrm{spk} / \mathrm{s}(\mathrm{mV} / \mathrm{cm}) ; 5$ for session 1 (18\%); 6 for session 2 (22\%); 0 for session $3(0 \%)$ ]; (3) chirp responders ["cr"; neurons that responded exclusively to the chirp (i.e., CSI $\geq 0.1$ and gain $<2 \mathrm{spk} / \mathrm{s}(\mathrm{mV} / \mathrm{cm}) ; 4$ for session $1(14 \%) ; 2$ for session $2(7 \%) ; 0$ for session $3(0 \%)$ ]; and (4) nonresponders ("nr"; neurons that neither responded to the beat nor the chirp (i.e., CSI $<0.1$ and gain $<2 \mathrm{spk} / \mathrm{s}$ $(\mathrm{mV} / \mathrm{cm})$; 3 for session 1 (11\%); 11 for session $2(41 \%) ; 0$ for session 3 $(0 \%)]$.

Computing signal and noise correlations. We quantified correlations between neuron activities from all neurons $(N=158)$ recorded during all three recording sessions using spike count sequences that were obtained from each binary spike train (i.e., unfiltered) by counting the 


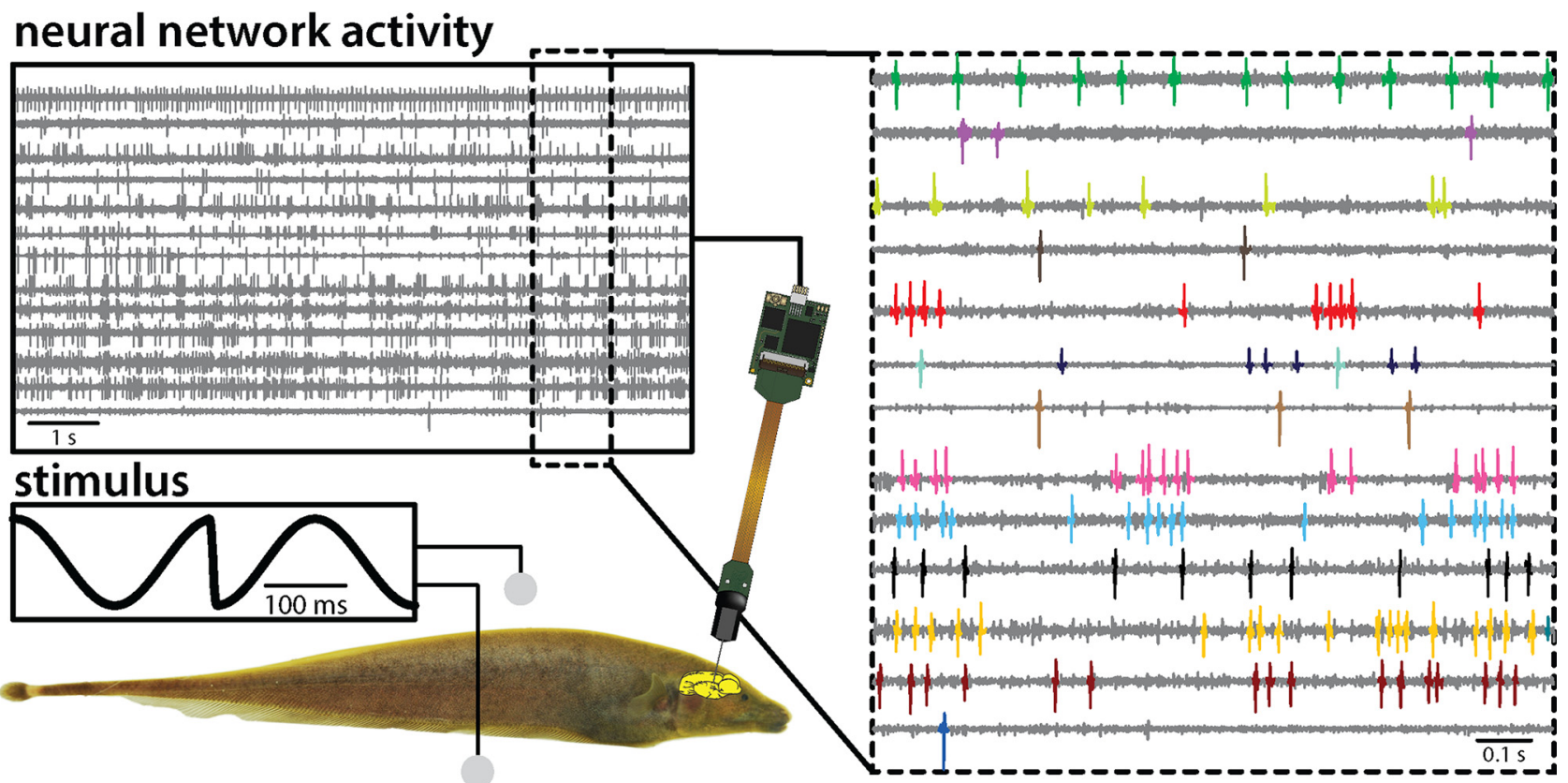

b
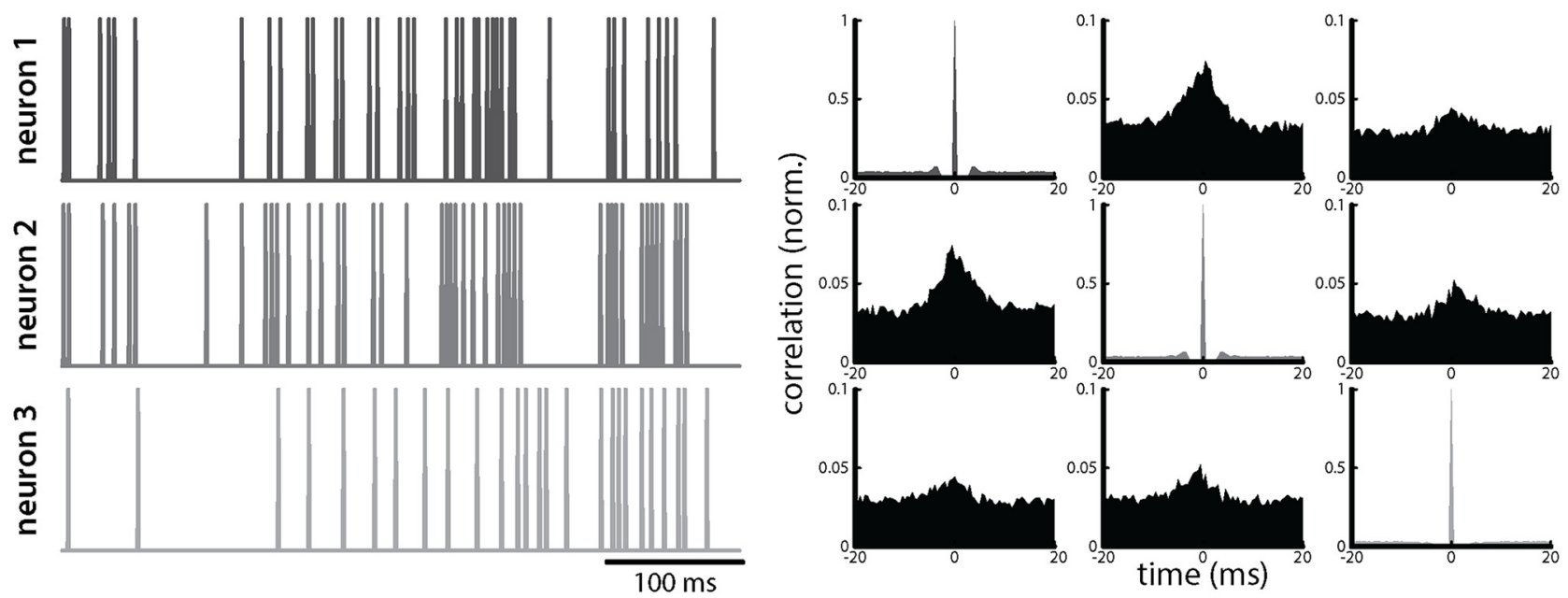

C stimulus

TS circuitry
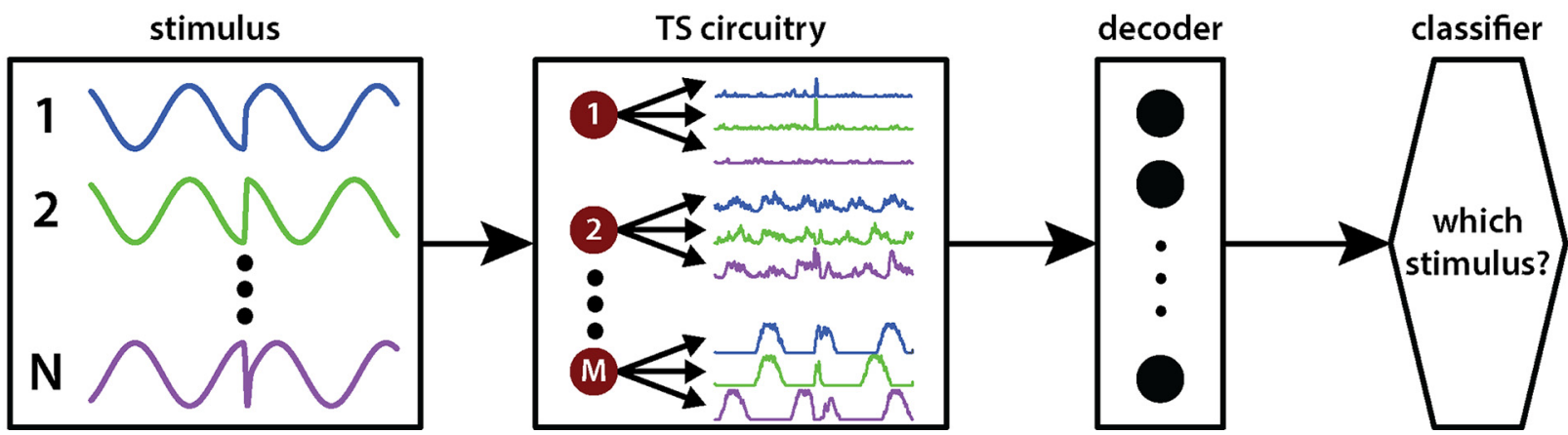

Figure 2. Methodologies used in this study. $\boldsymbol{a}$, Schematic showing the experimental setup. A fish is placed in an otherwise empty tank and is stimulated (middle left) while neuronal activity (top left and right) is recorded. $\boldsymbol{b}$, Left, Simultaneously recorded neurons display differential levels of baseline correlations as shown by the spike trains of three example neurons. Right, Autocorrelation and cross-correlation functions among these three different neurons. c, A set of electrocommunication stimuli with varying parameters ( $\mathrm{N}$, left) elicits differential responses in neurons within the midbrain torus semicircularis (TS, M, middle, left). These responses have to be decoded by downstream areas (middle, right) so as to optimally discriminate among the individual stimuli (right).

number of spikes occurring during successive and nonoverlapping $20 \mathrm{~ms}$ time windows that were always aligned with respect to the onset of the chirp stimulus during a $100 \mathrm{~ms}$ evaluation time window. This value was chosen because it comprises the neural response for most of the stimuli considered. In the case of larger durations, this time window comprises the initial portion of the response. We then computed the correlation coefficient between the two spike count sequences using Pearson's correlation coefficient between spike counts, as follows: 


$$
r_{\text {total }}=\frac{\operatorname{Cov}\left(n_{1} n_{2}\right)}{\sqrt{\operatorname{Var}\left(n_{1}\right) \operatorname{Var}\left(n_{2}\right)}} .
$$

To compute signal correlations, the 60 stimulus trials were first randomly permuted, and the spike count sequences were then recomputed (i.e., the timing of spikes within each trial is fixed). Signal correlations were then computed using Equation 3 on the shuffled spike counts and were averaged over 60 independent realizations of the shuffling procedure. Finally, noise correlations were computed as the correlation coefficient between the spike count residual sequences. The spike count sequences were first averaged over trials, and the mean spike count sequence (i.e., that because of the stimulus) was then subtracted from the spike counts for each trial to obtain the residual spike count sequences. As such, the residual spike count sequences represent the component of the neural response that cannot be explained by a given chirp stimulus waveform (i.e., "noise") because, unlike the stimulus waveform itself, these are not constant across trials.

Ascertaining statistical significance for obtained correlation coefficient values. The significance of correlations for each pair was determined in the following way. For each spike train, the sequence of interspike intervals (i.e., the times between consecutive action potentials) was obtained and then randomly shuffled using a permutation of the integers (i.e., the function "randperm" in MATLAB), and the spike times were then obtained by performing a cumulative sum (i.e., the function "cumsum" in MATLAB). Correlations were computed from the shuffled spike trains in otherwise the same manner as for the raw data as described above. The procedure was repeated 100 times to obtain the distributions of correlation coefficients. Correlation coefficients obtained from the raw (i.e., unshuffled) data were deemed to be significant if their probability of occurrence was $<0.05$ based on the distributions obtained from shuffled data. The mean shuffled signal and noise correlations for each possible pair for all distributions were not significantly different from zero (one-sample $t$ test, $\left.n=3572, p_{\text {signal }}>0.05 ; p_{\text {noise }}>0.05\right)$.

Classifier. We used a classifier to quantify the performance of TS neuronal populations at stimulus discrimination. We combined TS neurons using either equally or unequally weighted sums for each chirp stimulus. For each individual chirp stimulus, the combined response (i.e., either an equally or unequally weighted sum of neural responses) to a random trial was taken as the template for a given chirp stimulus waveform. Next, each combined response was assigned as being generated by the stimulus that gave rise to a given template based on whether the distance between the combined response and the template was the minimum. We thus constructed a "confusion matrix" whose element $(i, j)$ gives the probability that a response was assigned as being generated by stimulus $j$ given that it was actually generated by stimulus $i$ (Jamali et al., 2016, 2019). The diagonal elements of this matrix are the probabilities that a stimulus was correctly assigned, whereas nonzero off-diagonal elements indicate misclassification. For each confusion matrix obtained from the metric-space analysis, we computed the discrimination performance by averaging over the diagonal elements. The discrimination performance can thus vary between 0 (no discrimination) and 1 (perfect discrimination). Note that the chance level for discrimination performance was 0.0278 (i.e., 1 of 36) because we used a total of 36 different chirp stimuli. The distance between combined neuron activities was computed using the metric of van Rossum (2001). First, the combined neuronal activities were convolved with a decaying exponential kernel with time constant $\tau$, as follows:

$$
f(t)=\sum_{i=1}^{M} H\left(t-t_{i}\right) e^{\frac{-\left(t-t_{i}\right)}{\tau}}
$$

where $t_{i}$ is the $i$ th spike time, $M$ is the total number of spikes, and $H$ $(t)$ is the Heaviside step function $[H(10)=0$ if $x<0$ and $H(10)=1$ if $x \geq 0$ ]. Next, the distance was then computed as the euclidian distance between convolved combined neuronal activities $f R_{j}$ and $f R_{k}$, as follows:

$$
D\left(f_{R_{j}}, f_{R_{k}}\right)_{\tau}=\sqrt{\frac{1}{\tau} \int\left[f_{R_{j}}-f_{R_{k}}\right]^{2} d t}
$$

We varied $\tau$ between 1 and $100 \mathrm{~ms}$ to evaluate the effects of precise spike timing on classification. When the value of $\tau$ is small, the metric takes into account spike timing, whereas, when the value of $\tau$ is larger, the metric takes into account slower changes in firing rate. We also varied the chirp evaluation window between 20 and $1000 \mathrm{~ms}$ to evaluate the effects of the ongoing beat on discrimination performance (see Fig. 9). Performances reported in this article were averaged over templates, and error bars were generated over the total number of simulations for different $\tau$ and chirp evaluation windows (see Figs. $7 e, 8 c$, middle, bottom, 9) or by bootstrapping for different population sizes (i.e., 100 random chosen neuron responses per population size $k$ for $n_{k}<n$; see Figs. $7 d$, $8 b$, middle, bottom).

Differential evolution algorithm. To determine whether performing an unequally weighted sum of neuronal response gave rise to better classification than an equally weighted sum, we trained a differential evolution algorithm (EA) using the population responses on a randomly selected $60 \%$ of trials for each chirp stimulus as a training dataset. We then measured the classification accuracy of the trained classifier on the remaining $40 \%$ of trials (the test dataset). We chose recording session 2 for this analysis since it contained the greatest number of neurons recorded simultaneously that received all 36 stimuli $(n=27)$.

Specifically, each neuron was assigned a "weight," which could vary between -2 and 2 , and the goal was to choose a set of weights that maximizes the performance of the classification algorithm described above. The EA was described in detail in a previous study by our group (Aumentado-Armstrong et al., 2015). Specifically, a set of weight vectors (i.e., "agents") is allowed to evolve by minimizing a fitness function $F_{\text {fit }}$ over a series of iterations (i.e., "generations"). In keeping with the notation used in previous studies (Aumentado-Armstrong et al., 2015), we denote $X_{k}^{r}(i)$ as parameter $i$ for agent $r$ of generation $k$. First, the population of $k$ individuals is randomly initialized with weight values that are uniformly distributed with zero mean and restrained between -2 and 2 . For each individual at every generation, a new individual is constructed by two operations consisting of "differentiation" and "recombination." In differentiation, the $r^{\text {th }}$ new parameter vector $X_{k \text {,trial }}^{r}$ is built by combining three other individuals $X_{k}^{r_{1}}, X_{k}^{r_{2}}$, and $X_{k}^{r_{3}}$, where $r_{1} \neq r_{2} \neq r_{3}$, as follows:

$$
X_{k, \text { trial }}^{r}=X_{k}^{r_{1}}+\left(X_{k}^{r_{2}}-X_{k}^{r_{3}}\right) F \forall r=1, \ldots, N
$$

where the differential weight $F=0.5$, and the three individuals are chosen based on a probability distribution that is preferentially weighted for more fit (i.e., lower fitness score) individuals, as follows:

$$
p_{k}^{r_{1}}=\lambda \exp \left(\frac{-F_{f i t}\left(X_{k}^{r_{i}}\right)}{\max _{\forall j}\left(1-F_{f i t}\left(X_{k}^{j}\right)\right)}\right) \forall r_{i}=1, \ldots, N,
$$

where $\lambda$ is a normalization constant such that the sum of probability values is equal to 1 . Recombination is then performed as follows:

$$
X_{\mathrm{mut}}^{r}(i)=\left\{\begin{array}{c}
X_{k, \mathrm{trial}}^{r}(i), \text { ifu }<C R \\
X_{k}^{r}(i), \text { otherwise }
\end{array} \forall r=1, \ldots, N ; i=1, \ldots, D,\right.
$$

where $u$ is a random variable generated from a uniform distribution $U$ $(0,1)$ and with crossover probability $\mathrm{CR}=0.9$. Selection is finally performed to produce the next generation via:

$$
X_{k+1}^{r}=\left\{\begin{array}{c}
X_{\text {mut }}^{r}, i f F_{f i t}\left(X_{\text {mut }}^{r}\right)<F_{f i t}\left(X_{k}^{r}\right) \\
X_{k}^{r}, \text { otherwise }
\end{array} \forall r=1, \ldots, N .\right.
$$

In this study, the fitness function for a given individual was defined as follows: 


$$
F_{\text {fit }}\left(X_{k}^{r}\right)=1-D P_{X_{k}^{r}}
$$

where $D P_{X_{k}^{r}}$ is the discrimination performance estimated by computing the precision of events (i.e., spikes) of our neuronal population in response to our set of 36 chirp stimulus waveforms. The EA was terminated if the change in population discrimination performance for the previous 10 iterations was on average below a threshold value of 0.00001 .

We repeated the EA 20 times over different initial conditions to test whether different sets of weights would give rise to similar discrimination performance values. Based on the above, it is clear that if a given weight vector gives rise to a given performance, then the set of weight vectors obtained by multiplying the initial one by any nonzero constant will also give rise to the same performance. Thus, there is degeneracy because there exists an infinite number of weight vectors that will give rise to the same performance. Such "trivial" degeneracies were eliminated by normalizing weight vectors by their SDs and, if necessary, further multiplied by -1 when testing whether different weight vectors gave rise to a given performance (see Fig. 11).

Principal component analysis. Weight matrices obtained from the 20 EA simulations for a neuronal population with and without noise correlations were compared using principal component analysis (pca). Therefore, the sorted weight matrices were diagonalized using the singular value decomposition function in MATLAB. Trajectories were computed using the pca space of the first three principal components, explaining $>60 \%$ of the variance of the 20 sorted EA simulations. It is important to note that we used the same decomposition (i.e., linear combination of weights) to visualize weight vectors obtained with and without noise correlations. Therefore, any difference between the principal components has to be because of existing differences between the weight vectors. We compared the difference of the principal components with and without noise correlations with the difference of either with or without noise correlations obtained by bootstrapping (15 bootstraps with each randomly taking $75 \%$ of the total weight vectors) using the euclidian distance, as follows:

$$
d(p, q)=\sqrt{\left(p_{1}-q_{1}\right)^{2}+\left(p_{2}-q_{2}\right)^{2}+\left(p_{3}-q_{3}\right)^{2}}
$$

where $(p, q)$ are the Cartesian coordinates of the trajectories with and without noise correlations in three dimensions.

Response trial-to-trial variability. To quantify the response trial-totrial variability of the summed (i.e., either equally or unequally weighted) population activity to a given chirp stimulus waveform with and without noise correlations (equally weighted and weighted), we used the distribution within the interquartile range in responses averaged over the $20 \mathrm{EA}$ simulations, as follows:

$$
\text { variability }=\frac{\sum_{i=1}^{m} \sum_{j=1}^{n} D_{\mathrm{Q}_{2}}^{\mathrm{Q}_{3}} \sigma\left(k_{m, n}\right)}{m},
$$

where $D$ is the distribution within the interquartile range $\left(\mathrm{Q}_{2}=0.25 ; \mathrm{Q}_{3}\right.$ $=0.75), \sigma$ is the $\mathrm{SD}$ of the response ( $k$; trial-to-trial variability) to each stimulus $(m)$ and each EA simulation $(n)$. All responses were normalized before computing the response variability.

Similarity index. To quantify the similarity between individual responses $\left(x_{1}, x_{2}\right)$ to the set of stimulus waveforms used, we computed the similarity index (SI), which is a distance metric to assess the similarity between a response to a given stimulus to the responses to all stimulus waveforms that was computed as follows (Aumentado-Armstrong et al., 2015; Metzen et al., 2016, 2020; Metzen and Chacron, 2017):

$$
\operatorname{SI} x, y=\frac{\sqrt{\left\langle\left(x_{1}-\left\langle x_{1}\right\rangle-x_{2}+\left\langle x_{2}\right\rangle\right)^{2}\right\rangle}}{\max \left[\frac{\max \left(x_{1}\right)-\min \left(x_{1}\right)}{\sqrt{2}}, \frac{\max \left(x_{2}\right)-\min \left(x_{2}\right)}{\sqrt{2}}\right]},
$$

where $\langle\ldots>$ denotes an average over an evaluation window of $100 \mathrm{~ms}$ after chirp onset that is shown as a blue band in the figures. All responses were normalized before computing SI. The SI measure was used to assess how different the neural responses to a given stimulus waveform are to the responses to all other stimulus waveforms (see Fig. 10), which relates to the discriminability between neural responses to different stimulus waveforms.

Statistics. Values are reported as the mean \pm SD. Statistical tests were performed using a one-way ANOVA with Bonferroni correction, unless otherwise stated.

\section{Results}

Here we investigated for the first time how TS neuronal populations encode natural electrocommunication stimuli with highly heterogeneous attributes. Under natural conditions, chirps occur during social interactions in which the emitter fish sends the signal to the receiver fish (Fig. 1a, top). This signal consists of a transient increase in the EOD frequency of the emitter fish with a given time duration and amplitude (i.e., the amount by which the EOD frequency increases; Fig. $1 a$, middle). Interactions between the EOD frequencies of the two fish give rise to a background beat that consists of a sinusoidal AM of the EOD (Fig. $1 a$, bottom, dashed gray). When considering the stimulus sensed by the receiver fish, the chirp signal not only transiently perturbs the beat at a given phase (Fig. $1 a$, bottom, blue) but also causes a phase advance of the beat (Fig. 1a, bottom, compare solid black curves, dashed gray curves). As such, chirps with different attributes give rise to very different AM stimulus waveforms (Fig. 1b). We systematically varied multiple chirp attributes such as the amplitude (i.e., the amount by which the EOD frequency increases; Fig. $1 b$, top left), the beat phase at which the chirp occurs (Fig. 1b, bottom left), and the duration of the frequency increase (Fig. 1b, right). We recorded the simultaneous activities of TS neurons in response to chirp stimuli that were delivered via a pair of electrodes located on either side (Fig. $2 a$ ). The use of a high-density array allowed us to record the activities of up to 46 TS neurons simultaneously (Fig. $2 a$, right), and we found that TS neuronal activities were correlated in the absence of stimulation (Fig. 2b). We thus used chirps with different waveforms occurring on top of a sinusoidal waveform to understand how the activities of a neuronal population are integrated to distinguish between different stimuli (Fig. 2c).

\section{Midbrain neuronal activities are correlated in the absence of stimulation}

We first characterized correlations between the activities of midbrain neurons in the absence of stimulation since these are indicative of the presence of noise correlations during stimulation (Hofmann and Chacron, 2017). Overall, pairs of neurons that were recorded on probe sites that were located close to one another (Fig. $3 a$ ) tended to display higher baseline correlations in magnitude than pairs of neurons that were recorded on probe sites that were located farther apart (Fig. $3 b$ ). Indeed, baseline correlation magnitude decreased as a function of increasing physical distance when considering pairs with significant baseline correlations (Fig. $3 c$, green dots and light green dashed line; Pearson correlation coefficient: $\left.r=-0.18, p=1.73 * 10^{-6}\right)$. In contrast, for pairs with nonsignificant baseline correlations, its magnitude did not decrease significantly as a function of distance (Fig. 3c, gray dots and black dashed line; Pearson correlation coefficient: $r=0.002, p=0.95)$. However, when considering all possible pairs (i.e., with significant and nonsignificant baseline correlation magnitudes), the decrease in baseline correlation magnitude remains significant (Pearson correlation coefficient: $r$ $\left.=-0.14, p=3.40 * 10^{-8}\right)$. As mentioned above, the presence of 
baseline correlations is important as these can be seen as the limit toward which noise correlations tend as stimulus intensity goes to zero (Hofmann and Chacron, 2017). As such, we expected that TS neurons will display noise correlations under stimulation, which will impact population coding, as described above.

\section{Midbrain neurons display signal and noise correlations under stimulation} The responses of single TS neurons in our dataset were highly heterogeneous and were similar to those described previously (Vonderschen and Chacron, 2011; Sproule et al., 2015). Overall, we found that neuronal responses to chirp stimuli were of four types, as shown in Figure $4 a$ (see Materials and Methods for details on the classification). Some TS neurons responded exclusively to the chirp and not at all to the beat (Fig. $4 a$, red), and were termed cr; others instead responded to both the chirp and the beat (Fig. $4 a$, purple) and were termed bcr; and yet other neurons in a separate subset only responded to the beat (Fig. $4 a$, blue) and were termed br. Consistent with previous results (Vonderschen and Chacron, 2011; Sproule et al., 2015), some TS neurons did not respond to either the chirp or the beat (Fig. $4 a$, yellow) and were termed nr. Previous studies have shown that such neurons tend to respond to other behaviorally relevant stimuli such as moving objects or envelopes (Vonderschen and Chacron, 2011; Sproule et al., 2015). Response latencies of the first spike occurring after stimulus onset (Fig. $4 b$, left) were highly heterogeneous and ranged from a few milliseconds to $100 \mathrm{~ms}$ (Fig. $4 b$, right), with bcr neurons having the shortest latencies on average (Fig. $4 b$, right inset; nr: $46.19 \pm 15.88 \mathrm{~ms}$; br: $34.02 \pm$ $16.67 \mathrm{~ms}$; bcr: $19.84 \pm 12.54 \mathrm{~ms}$; cr: 38.12 $\pm 16.36 \mathrm{~ms}$; one-way ANOVA: $\mathrm{df}=3$, $F=52.66 ; p_{\mathrm{nr}, \mathrm{br}}=1.98 * 10^{-6} ; p_{\mathrm{nr}, \mathrm{bcr}}=2.35$ $* 10^{-26} ; p_{\mathrm{nr}, \mathrm{cr}}=0.008 ; p_{\mathrm{br}, \mathrm{bcr}}=3.75 *$ $10^{-11} ; p_{\mathrm{br}, \mathrm{cr}}=0.42 ; p_{\mathrm{bcr}, \mathrm{cr}}=4.75 * 10^{-15}$; Bonferroni corrected).

We next looked at the correlation structure of TS neurons under stimulation [i.e., the relationship between signal correlation coefficient $\left(r_{\text {signal }}\right)$ and noise correlation coefficient $\left.\left(r_{\text {noise }}\right)\right]$. As mentioned above, looking at the correlation structure is important because the sign relationship (i.e., whether they have the same sign or have opposite signs) between signal and noise correlations strongly influences whether correlations are beneficial or detrimental to information transmission (Averbeck et al., 2006). Specifically, if signal and noise correlations are either both positive or negative, then this will reduce information. In contrast, if signal correlations are positive and for a timescale of $20 \mathrm{~ms}$.
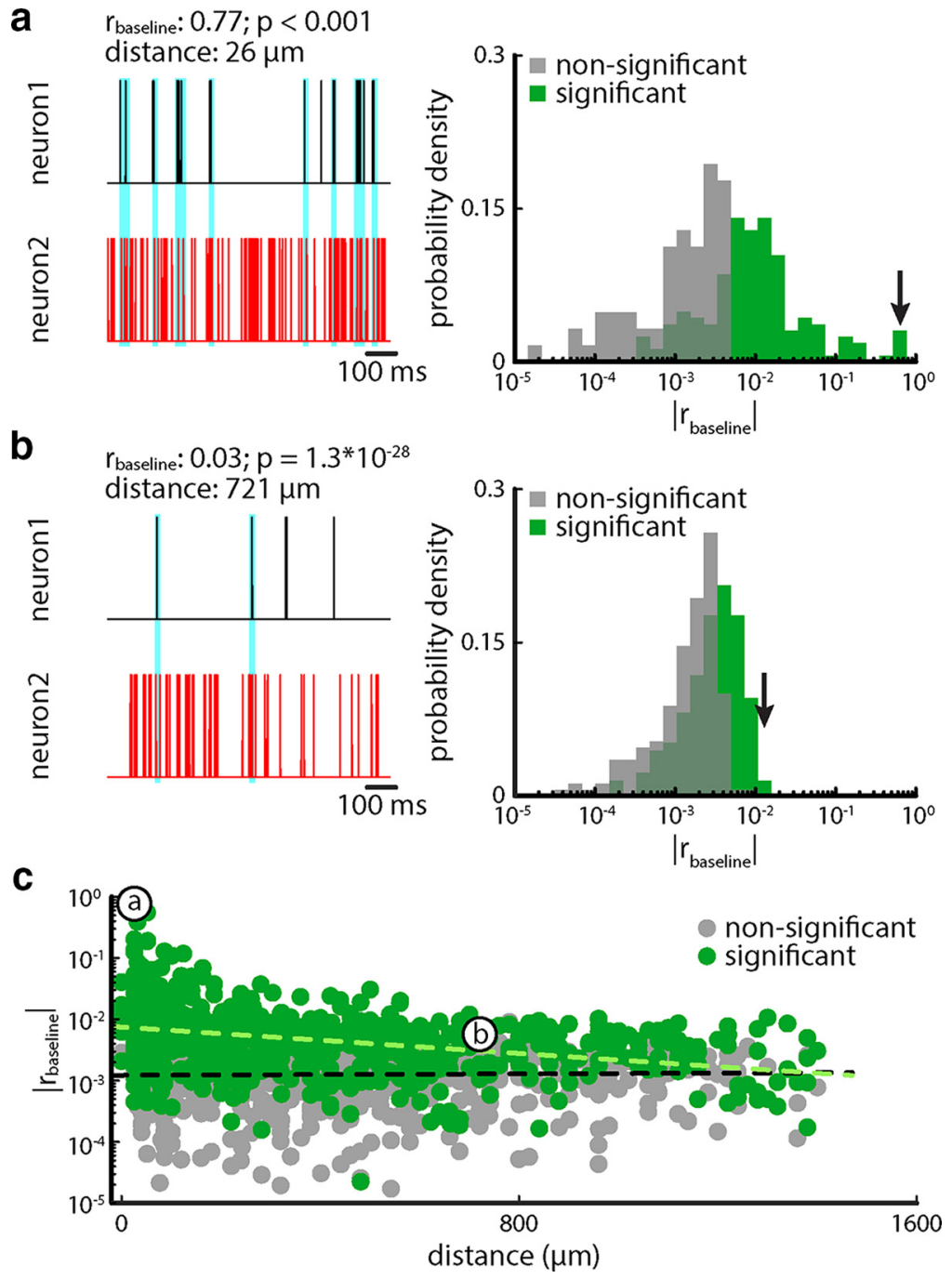

Figure 3. Baseline correlations decrease with increasing distance. $\boldsymbol{a}$, Left, Spiking activity for two example neurons (neuron 1, black; neuron 2, red) recorded on probe sites that were located close to one another $(26 \mu \mathrm{m})$. This pair displayed a large baseline correlation coefficient, as evidenced from coincident spiking that occurred more often than expected by chance (cyan bars). Right, Histogram of baseline correlation magnitude for all pairs $(n=120)$ for which the distance was $<100 \mu \mathrm{m}$. Shown are values significantly different from zero (green) and those that are not (gray). The black arrow indicates the value of correlation for the example shown in the left panel. $\boldsymbol{b}$, Left, Spiking activity for two example neurons (neuron 1, black; neuron 2 , red) recorded on probe sites that were located far from one another $(721 \mu \mathrm{m})$. This pair displayed a baseline correlation coefficient near zero, as evidenced from coincident spiking that occurred as often as expected by chance (cyan bars). Right, Histogram of baseline correlation magnitude for all pairs $(n=295)$ for which the distance was $\geq 700 \mu \mathrm{m}$. Shown are values significantly different from zero (green) and those that are not (gray). The black arrow indicates the value of correlation for the example shown in the left panel. c, Baseline correlation magnitude decrease as a function of distance (Pearson correlation coefficient: $r=-0.24, p=1.02 * 10^{-20}$ ). Shown are values significantly different from zero (green) and those that are not (gray). The labeled circles indicate the two examples shown in $\boldsymbol{a}$ and $\boldsymbol{b}$. Throughout, correlations were computed

noise correlations negative or vice versa, they will increase information transmission.

In general, signal correlations are correlations that are induced by two neuron populations receiving a common signal (Fig. 5a). We found that signal correlations across our datasets ranged between -0.73 and 0.84 , with mean magnitudes of 0.07 \pm 0.09 . Interestingly, $r_{\text {signal }}$ magnitudes decreased with increasing physical distance for pairs consisting of all response classes except "bcr-bcr" and "cr-cr" pairs (Fig. 5b; Pearson correlation coefficient between $r_{\text {signal }}$ and distance: bcr-bcr pairs: -0.16 , $p=0.17$; cr-cr pairs: $-0.51, p=0.07$ ). A similar relationship was seen when only considering neuron pairs whose correlation 


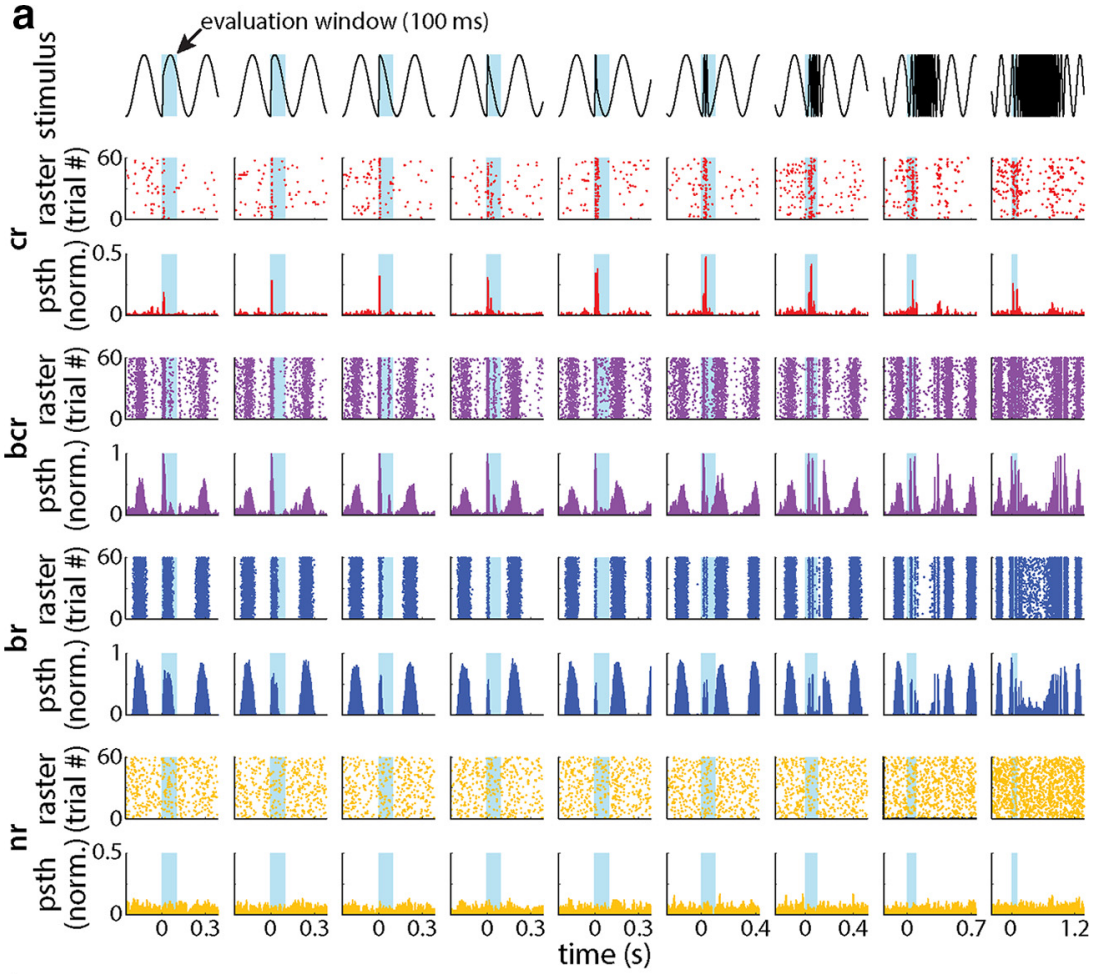

b

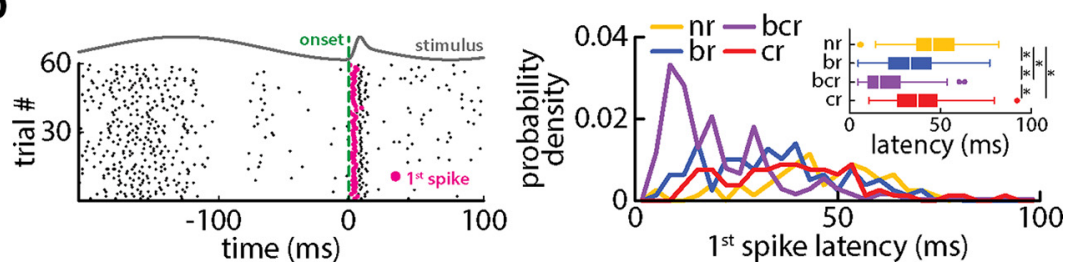

Figure 4. TS single neuronal responses to chirp stimuli are highly heterogeneous. $\boldsymbol{a}$, Raster plots and time-depend(red) strongly responded to the chirp waveform in a more or less invariant manner but not at all to the beat. The second (purple) responded to the chirp waveform but also phase locked to the beat. As such, the phase reset of the beat because of the chirp waveform also altered the response of this neuron. The third neuron (blue) responded to the beat but not to the chirp. The fourth neuron (yellow) did not respond to either the beat or the chirp. $\boldsymbol{b}$, Left, Schematic of how response latencies were estimated for the first spike (magenta) occurring after a chirp onset (green dotted vertical line). Right, Probability distributions of the first spike latencies of the four neurons response classes (nr, yellow; br, blue; bcr, purple; $\mathrm{cr}$, red). Inset, Boxplots showing the spike latencies obtained from the four response classes. The first spike latencies for bcr neurons are significantly lower than those of all other response classes (right inset; one-way ANOVA: $d f=3$, $F=52.66, p_{\mathrm{bcr}, \mathrm{nr}}=2.35 * 10^{-26}, p_{\mathrm{bcr}, \mathrm{br}}=3.75 * 10^{-11}, p_{\mathrm{bcr}, \mathrm{rr}}=4.75 * 10^{-15}$, Bonferroni corrected). ent firing rate responses of four example midbrain neurons to different chirp waveforms (shaded box). The first neuron

Materials and Methods), $r_{\text {signal }}$ significantly decreases (0.05 \pm 0.06 ; one-way ANOVA; $\mathrm{df}=3, F=27.74, p=8.12 * 10^{-17}$, Bonferroni corrected), while $r_{\text {noise significantly increases }}$ $(0.07 \pm 0.06$; one-way ANOVA: $\mathrm{df}=3$, $F=27.74, p=0.02$, Bonferroni corrected) with $r_{\text {signal }}$ and $r_{\text {noise }}$ being significantly different from each other (one-way ANOVA: $\mathrm{df}=3, F=27.74$, $p=5.55 * 10^{-14}$, Bonferroni corrected). Furthermore, we found no significant relationship between $r_{\text {noise }}$ and physical distance for all but br-br pairs (Fig. $5 f$; Pearson correlation coefficient between $r_{\text {noise }}$ and distance: "br-br" pairs: $\left.-0.15, p=3.7 * 10^{-6}\right)$. A similar relationship was seen when only considering neuron pairs that have correlation values significantly different from zero (Pearson correlation coefficient between $r_{\text {signal }}$ and distance: br-br pairs: $-0.16, p=3.49 * 10^{-6}$ ). In general, $r_{\text {noise }}$ magnitude was on average similar for pairs with small physical distances (Fig. $5 f, g ;<100 \mu \mathrm{m} ; 0.07 \pm 0.06)$ and with large physical distances (Fig. $5 f, h ; \geq 700 \mu \mathrm{m} ; 0.06$ $\pm 0.05)$. This is most likely because of the presence of long-range connections both within and across TS layers (Carr et al., 1981; Carr and Maler, 1985, 1986). To better illustrate this result, we computed the residuals of neuronal responses to chirp stimuli, which are the components of neuronal responses that cannot be explained by the stimulus (see Materials and Methods). Indeed, residuals for neuron pairs that are close to one another (Fig. $5 g$ ) or farther away from one another (Fig. 5h) were similarly correlated.

Overall, we found that signal and noise correlations were largely independent of chirp attributes. Indeed, signal and noise correlations were distributed in a similar manner for all chirp stimuli considered in the current study $\left(r_{\text {signal }}\right.$ : Kruskal-Wallis, $p=0.2784 ; r_{\text {noise: }}$ : Kruskal-Wallis, $p=0.99$ ). Figure $6 a$ shows the relationship between signal and noise correlations when varying chirp amplitude. Overall, signal and noise correlations were independent of one another (Pearson correlation coefficient between $r_{\text {signal }}$ and $r_{\text {noise }}$ : -0.014 ; values were significantly different from zero (Pearson correlation coefficient between $r_{\text {signal }}$ and distance: bcr-bcr pairs: -0.17 , $p=0.16$; cr-cr pairs: $-0.51, p=0.070$ ). In general, $r_{\text {signal }}$ magnitude was high on average for neurons that were in close proximity (Fig. $5 b, c ;<100 \mu \mathrm{m}, 0.09 \pm 0.13$ ) and low for pairs of neurons that were further apart (Fig. $5 b, d ; \geq 700 \mu \mathrm{m}, 0.04 \pm$ 0.04). Indeed, neurons that were located close to one another tended to display more similar responses to chirp stimuli than neurons that were located farther apart (Fig. 5, compare $c, d$ ).

When looking at noise correlations, which arise when two neuronal populations receive common input from a neuronal population that displays trial-to-trial variability to repeated stimulus presentations (i.e., "noise"; Fig. 5e), we found that these ranged between -0.50 and 0.74 with mean magnitudes of $0.06 \pm$ 0.05 . The magnitudes of $r_{\text {signal }}$ were significantly higher than those of $r_{\text {noise }}$ (one-way ANOVA: $\mathrm{df}=3, F=7.74, p=0.04$, Bonferroni corrected). However, when only considering pairs that have correlation values significantly different from zero (see $p=0.15$ ) with signal correlations ( $\mathrm{sc}_{\mathrm{tot}}$ ) being significantly higher than noise correlations $\left(\mathrm{nc}_{\mathrm{tot}}\right)$ on average for all possible pairs and significantly lower for neuron pairs that have correlation values significantly different from zero $\left(\mathrm{sc}_{\mathrm{resp}}, \mathrm{nc}_{\text {resp }}\right.$; see Materials and Methods; Fig. $6 b$; one-way ANOVA: $\mathrm{df}=3, F=32.16, p_{\text {tot }}=$ $9.93 * 10^{-5}, p_{\text {resp }}=9.68 * 10^{-14}$, Bonferroni corrected). Qualitatively similar results were obtained when varying chirp duration (Fig. $6 c$ : Pearson correlation coefficient between $r_{\text {signal }}$ and $r_{\text {noise: }}-0.014 ; p=0.06$; Fig. $6 d$ : one-way ANOVA: $\mathrm{df}=3$, $F=30.70, p_{\text {tot }}=0.005, p_{\text {resp }}=1.57 * 10^{-14}$, Bonferroni corrected) and chirp phase (Fig. 6e: Pearson correlation coefficient between $r_{\text {signal }}$ and $r_{\text {noise: }}-0.02 ; p=0.14$; Fig. $6 f$ : one-way ANOVA: $\mathrm{df}=3$, $F=33.97, p_{\text {tot }}=0.02, p_{\text {resp }}=3.55 * 10^{-16}$, Bonferroni corrected).

\section{Decoding information from linear summation of midbrain neuronal activities}

We next quantified the performance of TS neuronal populations at encoding chirps with different attributes and first considered 
a

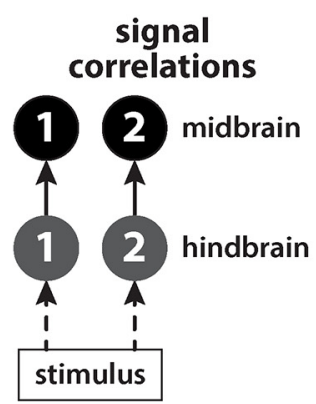

C

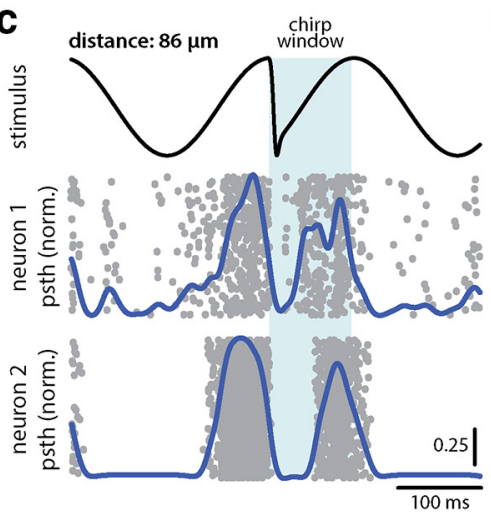

e

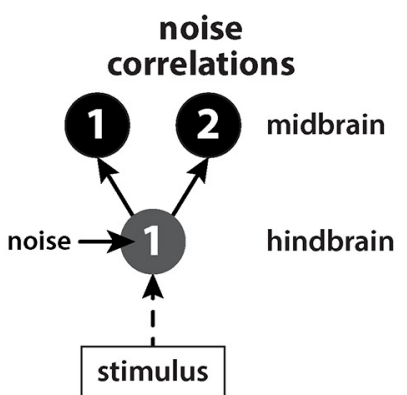

g
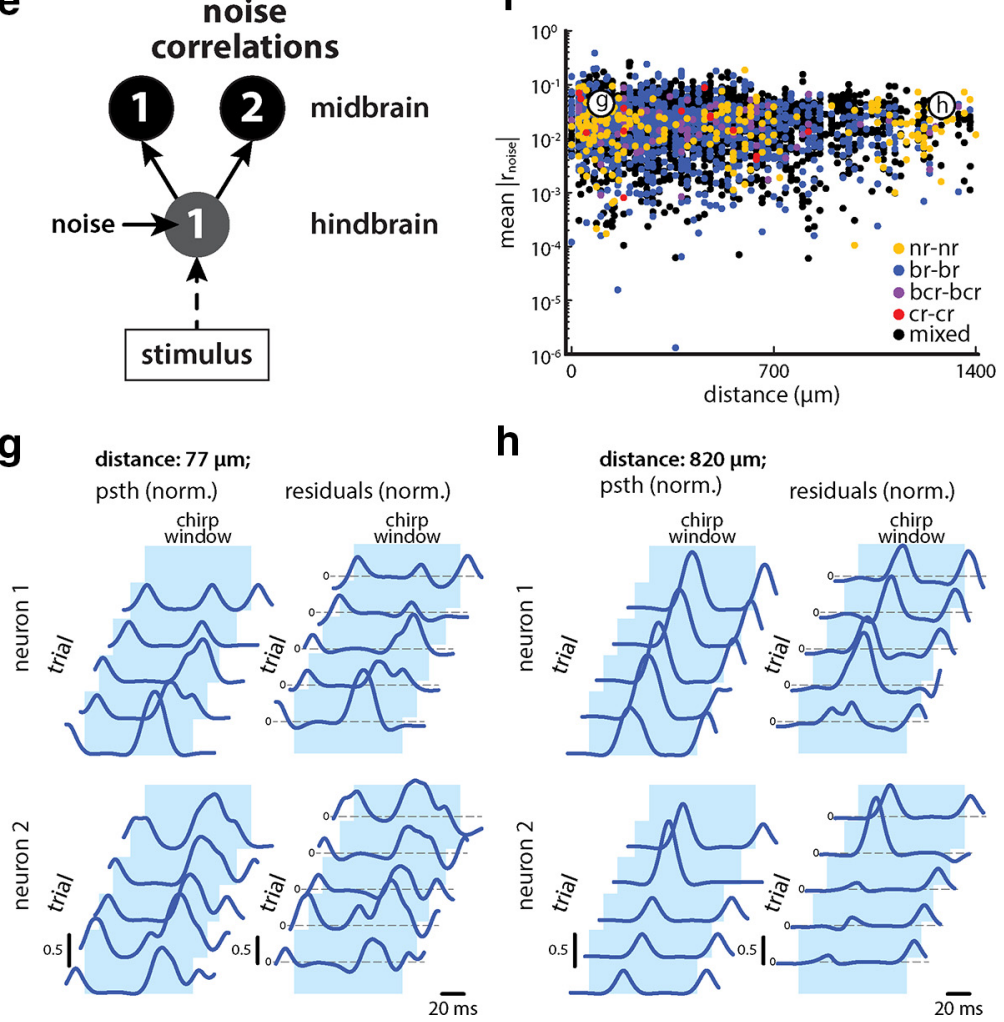

h
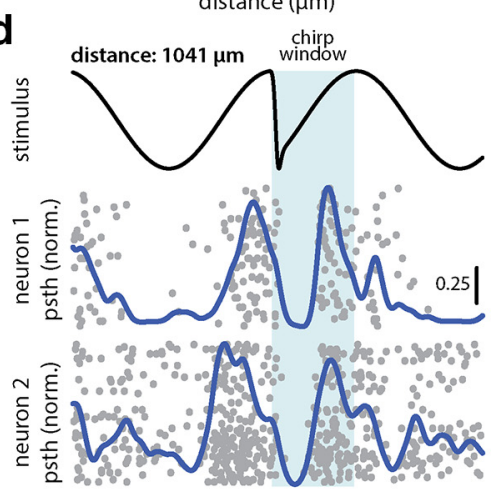

f
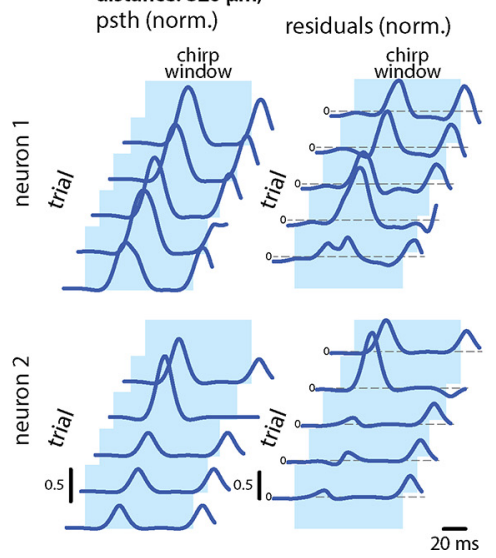

Figure 5. Signal correlations decrease with physical distance while noise correlations are independent of physical distance. $\boldsymbol{a}$, Schematic showing a hypothetical scenario in which a common stimulus is received by two afferent hindbrain neuron populations (gray circles). In this case, correlations between neuron populations "1" and "2" (black circles) will be exclusively because of the common stimulus and are referred to as signal correlations. $\boldsymbol{b}$, Signal correlation magnitudes are on average higher in magnitude between pairs with small physical distance $(\leq 100 \mu \mathrm{m})$ than in pairs with large physical distances $(>700 \mu \mathrm{m})$. The colored dots indicate the relation of signal correlation magnitudes and physical distance of same response type pairings ( $n r-n r$, yellow; br-br, blue; bcr-bcr, purple; $c r-c r$, red) as well as mixed pairings (black). The two labeled circles indicate the relation of signal correlation magnitudes and physical distance of the example pairs shown in $\boldsymbol{c}$ and $\boldsymbol{d}$. Each datapoint corresponds to one pair for one stimulus waveform. $\boldsymbol{c}$, An example pair of br TS neurons with small physical distance $(83 \mu \mathrm{m})$ displays a signal correlation of 0.53 in response to a given chirp stimulus (top, black) because their PSTHs are similar to each other (middle, neuron 1; bottom, neuron that the activities of TS neurons are summed linearly (i.e., equally weighted; Fig. $7 a$ ). Combined responses to different stimuli were compared using a distance metric (see Materials and Methods). Next, to determine whether a given response was correctly predicted as having been elicited by a given stimulus, the discrimination performance was computed from the confusion matrix whose element $i j$ gives the conditional probability that a response generated by stimulus $i$ is classified as being generated by stimulus $j$. The diagonal elements thus represent correct assignments while off-diagonal elements instead represent incorrect assignments. Thus, if our classifier displayed $100 \%$ correct performance, then the diagonal of the confusion matrix would be unity with all off-diagonal elements zero. As such, we quantified the performance by taking the average value of the diagonal elements (see Materials and Methods). To maximize performance, we would expect that the sets of responses elicited by different chirp stimulus waveforms would be as distant from one another as possible, while making the size of each set (i.e., the trialto-trial variability in the response) as small as possible (Fig. $7 b$ ).

To quantify the impact of noise correlations, we compared the performances of the equally weighted summed neuronal activities before

\section{$\leftarrow$}

2). The gray dots show the spiking activity of both neurons to repeated presentations of the chirp stimulus (top, black). $\boldsymbol{d}$, An example pair of br TS neurons with large physical distance $(1005 \mu \mathrm{m})$ displays a signal correlation of 0.19 in response to a chirp stimulus (top, black) because their PSTHs are more dissimilar to each other (middle, neuron 1; bottom, neuron 2). The gray dots show the spiking activity of both neurons to repeated presentations of the same chirp stimulus (top, black). $\boldsymbol{e}$, Schematic showing a hypothetical scenario in which a stimulus is received by one afferent neuron hindbrain population (gray circle) that also receives a noise input, which then projects to two TS neuronal populations "1" and "2" (black circles). In this case, correlations between neuronal populations "1" and "2" will not only be because of the stimulus (i.e., signal correlations), but will also be because of the noise received by the afferent neuronal population (i.e., noise correlations). $\boldsymbol{f}$, Noise correlation magnitudes are on average independent of the physical distance between neurons. The colored dots indicate the relation of noise correlation magnitudes and physical distance of same response type pairings ( $n r-n r$, yellow; br-br, blue; bcr-bcr, purple; $\mathrm{Cr}-\mathrm{Cr}$, red) as well as mixed pairings (black). The two labeled circles indicate the relation of noise correlation magnitudes and physical distance of the example pairs shown in $\boldsymbol{g}$ and $\boldsymbol{h}$. Each datapoint corresponds to one pair for one stimulus waveform. $\boldsymbol{g}$, An example pair of br TS neurons with small physical distance $(77 \mu \mathrm{m})$ displays a noise correlation of 0.28 because their residuals covary within the chirp window of $100 \mathrm{~ms}$ (top right, neuron 1; bottom right, neuron 2) as shown for five representative trials to a given chirp stimulus. Also shown are the PSTHs for the same trials (top and bottom left). $\boldsymbol{h}$, An example pair of br TS neurons with large physical distance $(820 \mu \mathrm{m})$ displays a noise correlation of 0.25 because their residuals covary within the chirp window of $100 \mathrm{~ms}$ (top right, neuron 1; bottom right, neuron 2) as shown for five representative trials to the same chirp stimulus. Also shown are the PSTHs for the same trials (top and bottom left). 
a

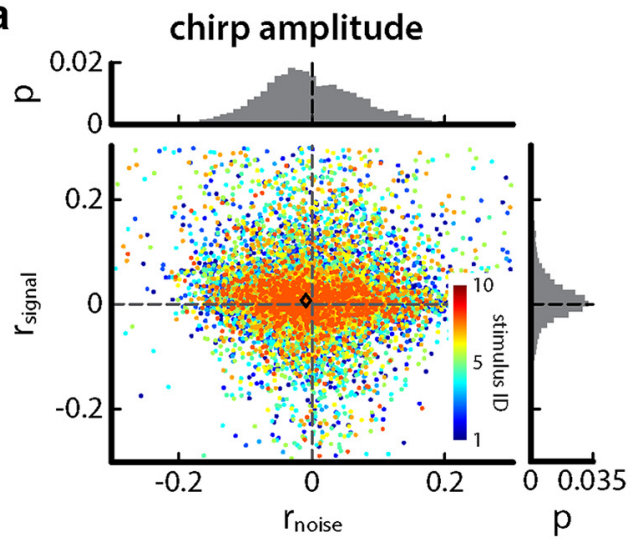

C

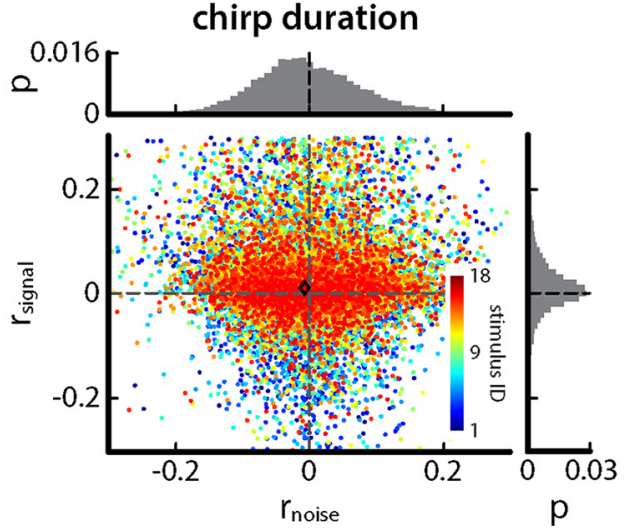

e

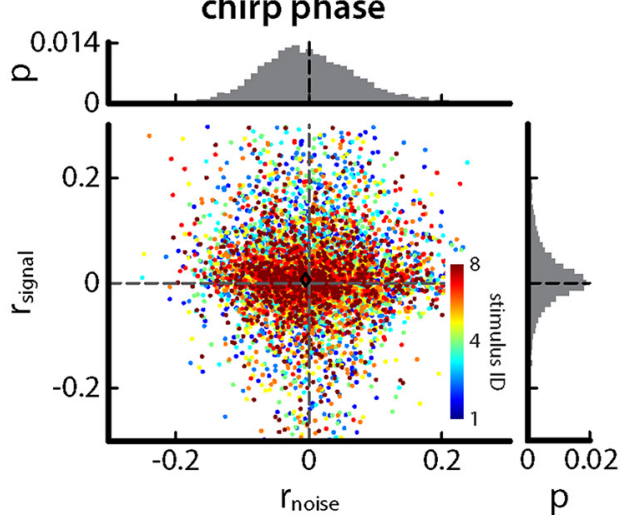

b

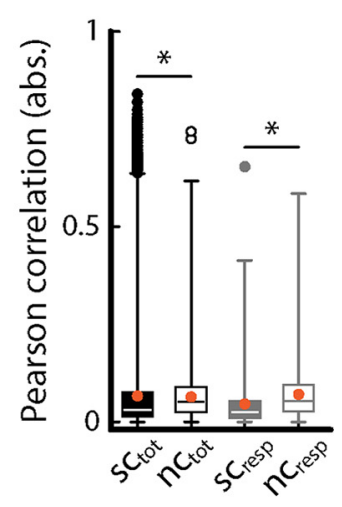

d

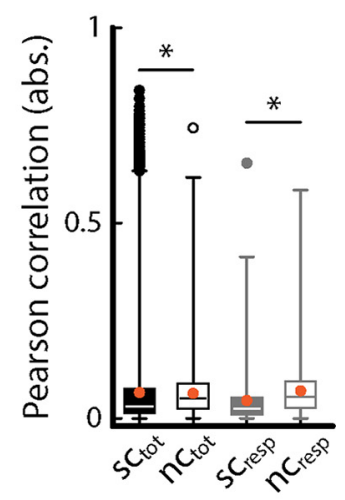

f

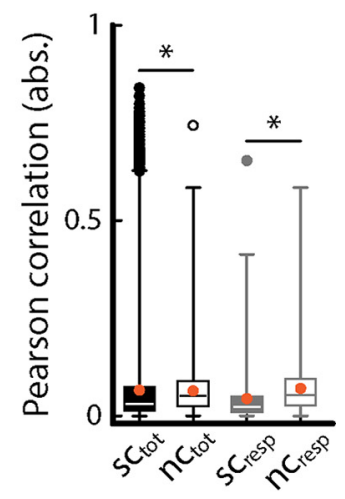

Figure 6. Noise and signal correlations are independent of the chirp and of one another. $\boldsymbol{a}$, Signal correlations as a function of noise correlations obtained when varying chirp duration. The color code indicates the individual stimulus IDs. The summed probability distributions for signal (right, gray) and noise (top, gray) are also shown. The diamond shows the mean signal and noise correlations. $\boldsymbol{b}$, Boxplot showing the magnitudes of signal (filled) and noise (hollow) correlation coefficients for all possible pairs (black) and for pairs that displayed correlation that have correlation values significantly different from zero. When considering all possible pairs, signal correlations were significantly higher than noise correlations but lower when considering pairs with correlation values significantly different from zero (one-way ANOVA: $d f=3$, $F=32.16, p_{\text {tot }}=9.93 * 10^{-5}, p_{\text {resp }}=9.68 * 10^{-14}$, Bonferroni corrected). The orange dots indicate the mean values. $\boldsymbol{C}$, Same as $\boldsymbol{a}$ but for chirp duration. $\boldsymbol{d}$, Boxplot showing the magnitudes of signal (filled) and noise (hollow) correlation coefficients for all possible pairs (black) and for pairs that have correlation values significantly different from zero. Signal correlations were significantly lower than noise correlations when considering all pairs or just pairs for which correlation coefficients were significantly different from zero (one-way ANOVA: $\mathrm{df}=3 ; F=30.70, p_{\text {tot }}=0.005, p_{\text {resp }}=1.57 * 10^{-14}$, Bonferroni corrected). The orange dots indicate the mean values. $\boldsymbol{e}$, Same as $\boldsymbol{a}$ but for chirp phase. $\boldsymbol{f}$, Boxplot showing the magnitudes of signal (filled) and noise (hollow) correlation coefficients for all possible pairs (black) and for pairs that have correlation values significantly different from zero. In each case, signal correlations were significantly lower than noise correlations (one-way ANOVA: $\mathrm{df}=3 ; F=33.97, p_{\text {tot }}=0.02, p_{\text {resp }}=3.55 * 10^{-16}$, Bonferroni corrected). The orange dots indicate the mean values.

and after randomly shuffling neuronal responses with respect to stimulus trials, which will eliminate noise correlations (see Materials and Methods). Indeed, we verified that the noise correlations computed after shuffling were not significantly different from zero (see Materials and Methods). Our results show that the variability in the equally weighted summed neuronal responses were significantly lower after shuffling (Fig. $7 c$ : oneway ANOVA: $\mathrm{df}=3, \quad F=1.12 * 10^{4}$, $p=1.57 * 10^{-7}$, Bonferroni corrected). This indicates that the variability in the responses can be better averaged away when noise correlations are not present (Zohary et al., 1994) and suggests that noise correlations have a detrimental effect with respect to discrimination performance overall. The top panel of Figure $7 d$ compares the confusion matrices obtained for different population sizes before (top) and after (bottom) randomly shuffling to eliminate noise correlations. Neuronal populations were constructed in the following way: first, $\mathrm{cr}$ neurons were considered, then bcr neurons were added, then br neurons, and finally $\mathrm{nr}$ neurons were added. In general, adding more neurons gave rise to better performance as values on the main diagonal of the confusion matrices increased overall (Fig. $7 d$, top) as quantified by increased performance (Fig. $7 d$, middle). Noise correlations had a detrimental effect on performance (Fig. $7 d$, middle inset: oneway ANOVA, $\mathrm{df}=3, F=721.3, p=2.38 *$ $10^{-13}$; Bonferroni corrected). Interestingly, the greatest improvement in performance was seen when bcr neurons and, to a lesser extent, "br" neurons were added to the population, while adding nonresponsive neurons actually decreased performance slightly (Fig. $7 d$, middle). This is further seen when comparing same-size populations of the different cell categories (Fig. $7 d$, bottom). As such, our results show that noise correlations, although overall small in magnitude, can have a significant effect on population coding by large neural populations, which is expected (Schneidman et al., 2006).

We also systematically varied the timescale used to compute the distance between spike trains (see Materials and Methods). Low values of timescale take precise spike timing into account while larger values take slower changes in firing rate (van Rossum, 2001; Jamali et al., 2016, 2019). Overall, maximum performance was observed for a timescale near $6 \mathrm{~ms}$ (Fig. 7e), indicating that information is contained in the precise spike timing of TS neurons. Overall, performances computed on the shuffled responses were significantly greater than those obtained before shuffling for all timescales tested (Fig. 
a
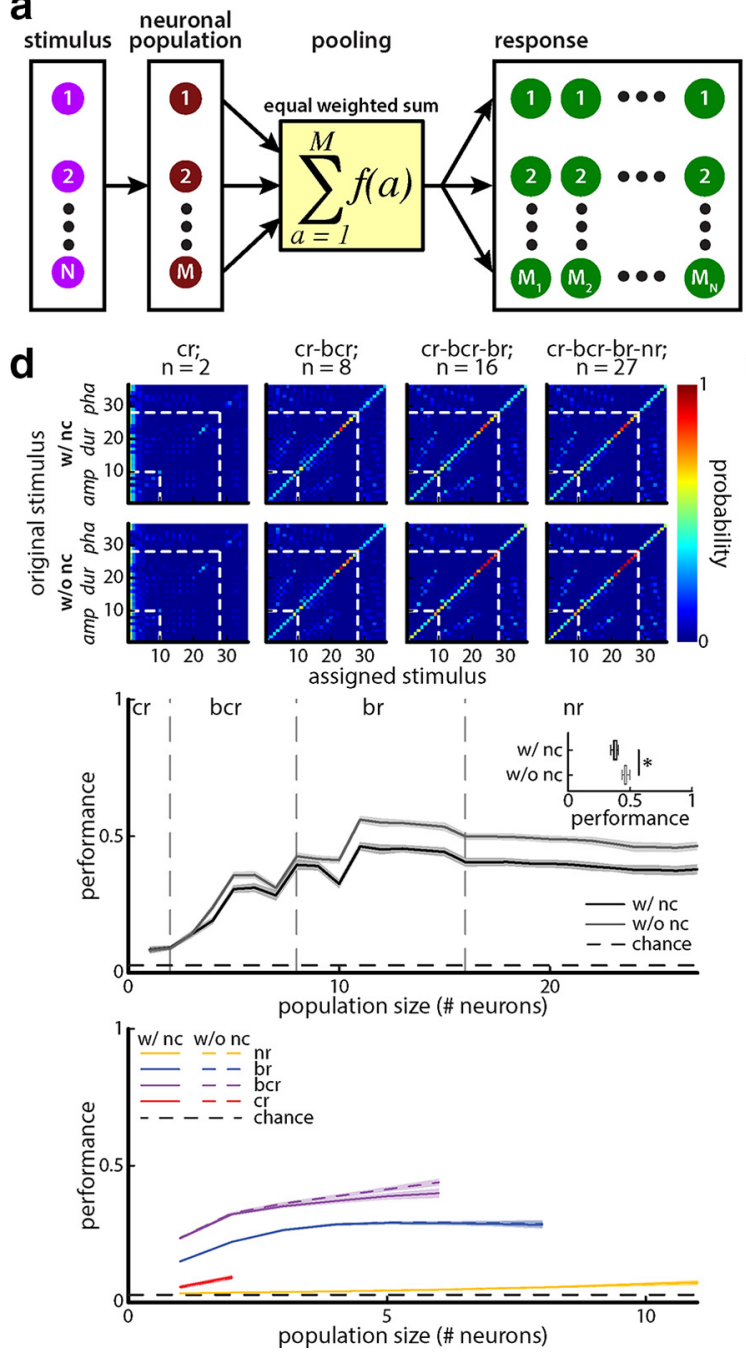

b
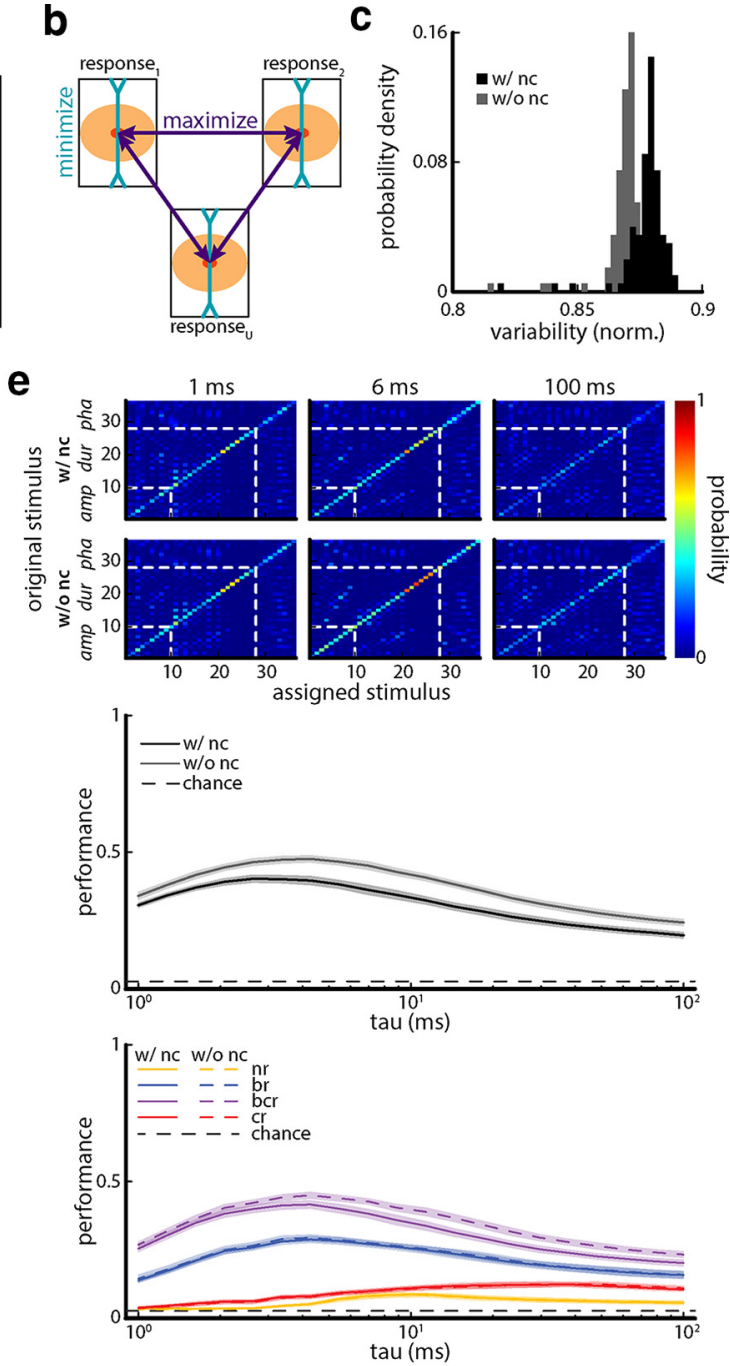

Figure 7. Noise correlations have detrimental effects on discrimination performance when taking the equally weighted sum of neuronal activities. $\boldsymbol{a}$, Schematic showing how the population response to each stimulus $\left(M_{1}-M_{N}\right)$ was obtained by summing the responses $(M)$ of each neuron to a given chirp stimulus for the equally weighted case. $\boldsymbol{b}$, Theoretical assumption illustrating that the population response variability to individual stimuli should be minimized while the response distances should be maximized to optimally discriminate among individual stimuli. $\boldsymbol{c}$, Variability probability densities with (black) and without (gray) noise correlations. The response variability to our 36 different chirp stimuli is significantly lower when noise correlations have been removed from our population by shuffling compared with the same population with noise correlations (one-way ANOVA: $\mathrm{df}=3 \mathrm{~F}=1.12 * 10^{4}, p=1.57 * 10^{-7}$, Bonferroni corrected). $\boldsymbol{d}$, Top, Confusion matrices showing the conditional probability of assessing an equally weighted population response to be caused by stimulus $i$ when it was actually caused by stimulus $j$ computed from metric-space analysis using the van Rossum measure with timescale $\tau=6 \mathrm{~ms}$ for population sizes of 2 (left), 8 (middle left), 16 (middle right), and 27 (right) neurons with (top) and without (bottom) noise correlations. The white dashed lines indicate borders between blocks of stimuli for which a given attribute (e.g., amplitude, duration, phase) was varied. Middle, Discrimination performance as a function of population size with (black) and without (gray) noise correlation for the equally weighted case. The dashed line indicates chance level (1 of 36 stimuli). Note that the jumps in performance occur when neurons with better discrimination performance are added. Inset, Performance with (black) and without (gray) noise correlations for population size of 27 were significantly different from one another (with noise correlations vs without noise correlations; one-way ANOVA: $\mathrm{df}=3, F=71.69, p=2.38 * 10^{-13}$, Bonferroni corrected). Bottom, Discrimination performance as a function of population size with (solid) and without (dashed) noise correlation for the equally weighted case when neuronal populations consisting only of neurons of a given type (i.e., $\mathrm{nr}$, br, bcr, and cr) are considered. $\boldsymbol{e}$, Top, Same as c, but now for a population size of $n=27$ neurons with (top) and without (bottom) noise correlations but for timescales of $1 \mathrm{~ms}$ (left), $6 \mathrm{~ms}$ (middle), and $100 \mathrm{~ms}$ (right). Middle, Discrimination performance of the equally weighted population as a function of timescale with (black) and without (gray) noise correlations. The dashed line indicates chance level (1 of 36 stimuli). Bottom, Discrimination performance as a function of timescale with (solid) and without (dashed) noise correlation for the equally weighted case when neuronal populations consisting only of neurons of a given type (i.e., $\mathrm{nr}, \mathrm{br}, \mathrm{bcr}$, and cr) are considered.

7 e, compare black curves, gray curves; $t$ test, $p<3.86 * 10^{-8}$ ). Interestingly, when computing performance, almost no cell category displayed a significant difference in performance with and without noise correlations, although the performance magnitude greatly varied across the different cell categories (Fig. 7e, bottom).

Performing an unequally weighted sum of TS neuronal activities gives rise to improved performance and eliminates the detrimental effects of noise correlations

We next asked whether assigning individual weights to each neuron would give rise to increased discrimination performance.
Weights were either positive or negative and could represent, for instance, the synaptic weights of connections to a downstream neuron. This question is particularly interesting here since signal and noise correlations were independent of one another. As such, theoretically, one should be able to combine neuronal activities so as to eliminate redundancy without compromising trial-averaged neuronal responses. Thus, we considered a decoder for which an unequally weighted sum of neuronal activities was performed (i.e., each neuron was assigned its own weight before summation; Fig. 8a). To find which combinations of weights (i.e., "weight vectors") gave rise to the best performance, 
a

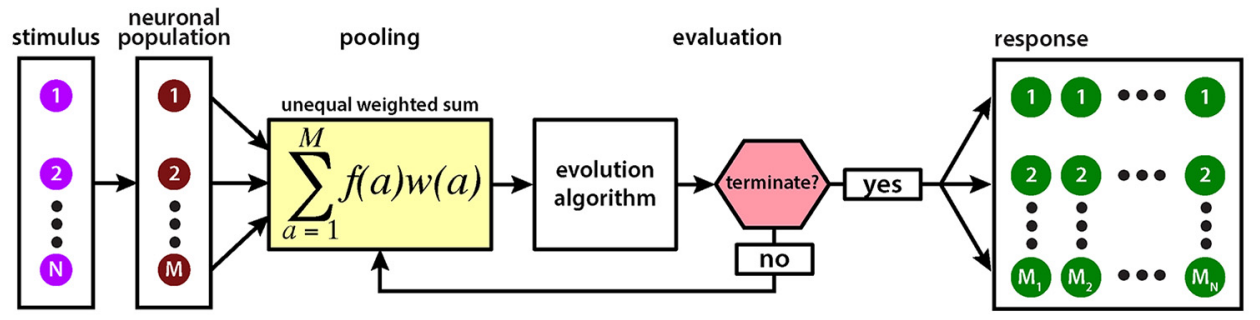

b

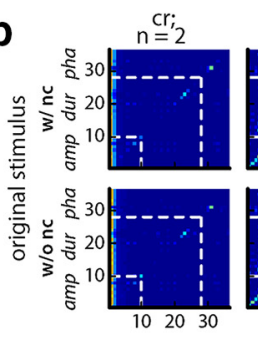

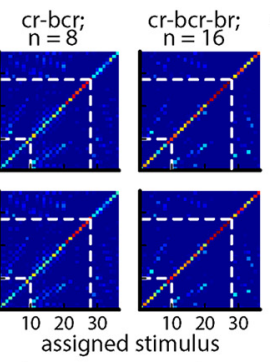
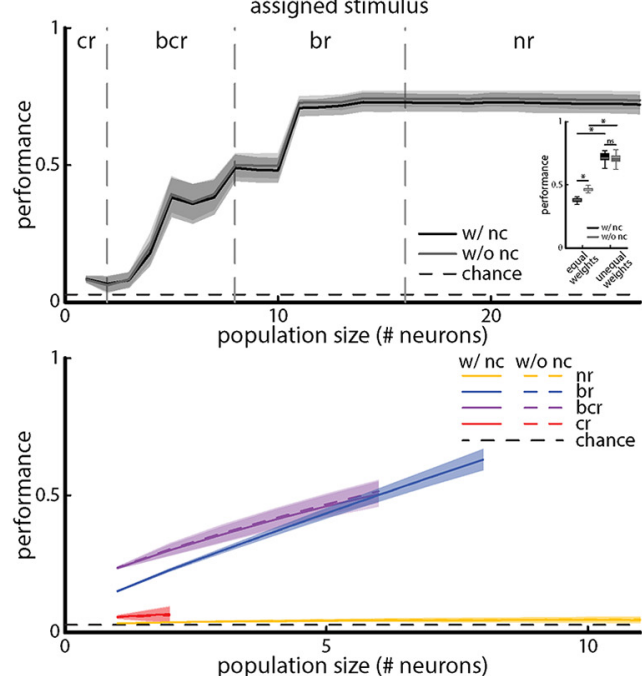

C
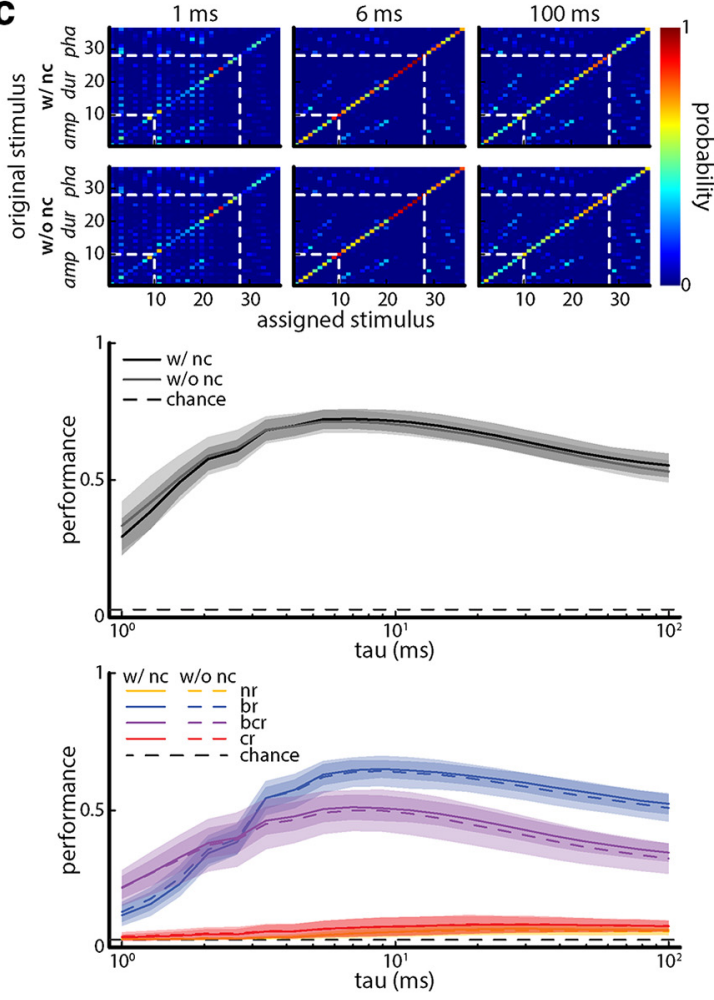

Figure 8. Taking an unequally weighted sum of neuronal activities greatly improves performance and mitigates the deleterious effects of noise correlations. $\boldsymbol{a}$, Schematic illustrating how individual weights were obtained by an iterative EA. First, each neuronal response ( $M$, left, dark red) to a given stimulus (left, pink) is weighted (middle, yellow) and its discrimination performance is compared with that of the previous iteration. If the discrimination performance is better, the respective weight value is kept; if not, a new weight value is generated by the EA (see Materials and Methods). This procedure is performed for each agent (middle). Finally, a population response to each stimulus ( $M_{1}-M_{N}$, right, green) is generated by summing the unequally weighted responses, and the discrimination performance of that population is assessed. $\boldsymbol{b}$, Top, Confusion matrices showing the conditional probability of assessing an unequally weighted neuronal population response as being caused by stimulus $i$ when it was actually caused by stimulus $j$ computed from metric-space analysis using the van Rossum measure with timescale $\tau=$ $6 \mathrm{~ms}$ for population sizes of 2 (left), 8 (middle left), 16 (middle right), and 27 (right) neurons with (top) and without (bottom) noise correlations. The white dashed lines indicate borders between blocks of stimuli for which a given attribute (e.g., amplitude, duration, phase) was varied. Middle, Discrimination performance as a function of population size with (black) and without (gray) noise correlation for the unequally weighted case. The dashed line indicates chance level (1 of 36 stimuli). Inset, Performance with (black) and without (gray) noise correlations for population size of 27 were not significantly different from one another in the unequally weighted case (one-way ANOVA: $d f=3, F=721.3, p=0.64$, Bonferroni corrected). Also shown are values obtained for the equally weighted case for comparison. Bottom, Discrimination performance as a function of population size with (solid) and without (dashed) noise correlation for the unequally weighted case when neural populations consisting only of neurons of a given type (i.e., nr, br, bcr, and cr) are considered. c, Top, Same as c, but for the same population size ( $n=27$ neurons) with (top) and without (bottom) noise correlations and for timescales of $1 \mathrm{~ms}$ (left), $6 \mathrm{~ms}$ (middle), and $100 \mathrm{~ms}$ (right). Middle, Discrimination performance as a function of timescale with (black) and without (gray) noise correlations. The dashed line indicates chance level (1 of 36 stimuli). Bottom, Discrimination performance as a function of timescale with (solid) and without (dashed) noise correlation for the unequally weighted case when neural populations consisting only of neurons of a given type (i.e., nr, br, bcr, and cr) are considered.

we used an EA (see Materials and Methods) that allowed weight vectors to "evolve" over "generations" (Fig. $8 a$; see Materials and Methods). Figure $8 b$ shows confusion matrices obtained with (top) and without (second top) noise correlations averaged over different realizations of the EA. It is important to note that the EA simulations were performed independently on the raw and shuffled datasets and that the best weight vectors obtained with and without noise correlations differed from one another. Overall, performances obtained using unequally weighted sums were significantly improved over those obtained using equally weighted sums (compare Figs. $8 b, 7 d$; Fig. $8 b$, middle inset: oneway ANOVA: $\mathrm{df}=3, F=721.3$; with noise correlations equally weighted vs unequally weighted, $p=3.86 * 10^{-50}$; without noise correlations equally vs unequally weighted, $p=1.20 * 10^{-39}$; Bonferroni corrected). Adding more neurons improved performance overall but the greatest improvement was seen when bcr and br neurons were added to the pool (Fig. $8 b$, middle). Adding nr neurons did not give rise to any improvement in performance but did not degrade performance. Interestingly, similar performances were obtained with and without noise correlations (Fig. 8b, middle, compare black curves, gray curves; one-way ANOVA: $\mathrm{df}=3, F=721.3, p=0.64$, Bonferroni corrected), confirming our prediction that the deleterious effects of noise correlations seen when considering linear sums could indeed by 
mitigated. This is further seen when comparing same-size populations of the different cell categories (Fig. 8b, bottom). Varying the timescale in our distance metric gave rise to qualitatively similar results as those seen using linear sums as best performance was still observed for a timescale of $6 \mathrm{~ms}$ (Fig. $8 c$ ). We also looked at the effects of increasing chirp evaluation windows on performance (Fig. 9). We found that larger chirp evaluation windows also led to higher performances of our TS neuron population (Fig. 9). This is not surprising because for larger chirp evaluation windows, a larger portion of the response to the chirp-induced phase reset can be considered in the discrimination procedure.

The greater performances obtained using unequally weighted sums suggest that the decoder can effectively better separate responses to different chirp waveforms as well as minimize trial-to-trial variability, so as to improve discriminability (Fig. 10a). To test this prediction, we looked at the trial-averaged equally and unequally weighted summed activities in response to different chirp waveforms. We found that, indeed, the trial-averaged unequally weighted summed activities were more discriminable from each other than the trial-averaged equally weighted summed activities as they displayed greater variation (Fig. 10b, compare error bands). Furthermore, variability was in general greater early after stimulus onset compared with late after stimulus onset (Fig. 10c). Moreover, when computing trial-to-trial variability, we found greater values for equally weighted summed activities than for unequally weighted summed activities (Fig. 10b,c). These results are summarized in Figure 10, $d$ and $e$. Specifically, the similarity index between the trial-averaged unequally weighted summed activities were significantly greater than those between the trial-averaged equally weighted summed activities (Fig. 10d; oneway ANOVA: $\mathrm{df}=3 ; F=71.69$; with noise correlations equally weighted vs unequally weighted, $p=3.19 * 10^{-4}$; without noise correlations equally weighted vs unequally weighted, $p=4.55 * 10^{-35}$; Bonferroni corrected). Moreover, trial-totrial variability was significantly lower in the unequally weighted case with noise correlations (Fig. 10e: one-way ANOVA: $\mathrm{df}=3 ; F=1.12 * 10^{4} ; p=0.0001$; Bon-jerroni corrected) and without noise correlations (Fig. 10e: one-way ANOVA: $\mathrm{df}=3$; $F=1.12 * 10^{4} ; p=1.20 * 10^{-311}$; Bonferroni corrected). Interestingly, while the similarity index in the unequally weighted case was significantly higher for without than with noise correlations (Fig. 10d: one-way ANOVA: $\mathrm{df}=3 ; F=71.69 ; p=3.55 * 10^{-17}$; Bonferroni a
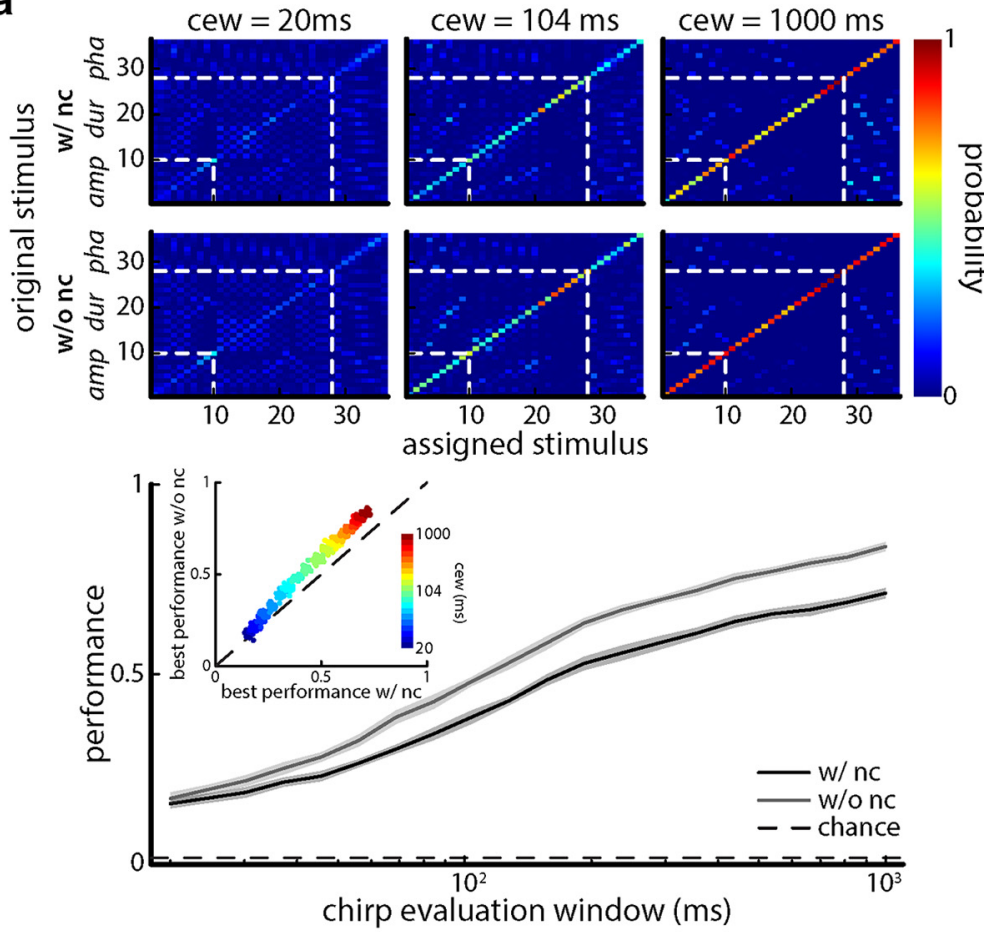

b
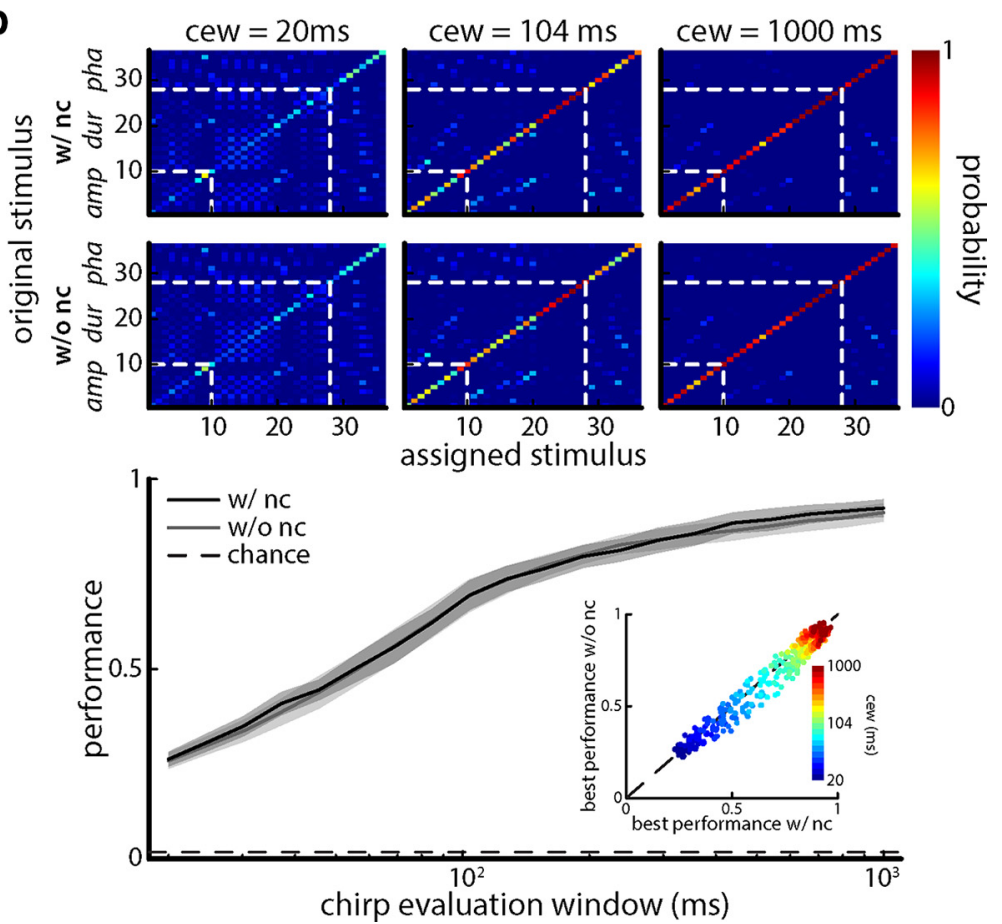

Figure 9. Performance increases for larger chirp evaluation windows. $\boldsymbol{a}$, Top, Confusion matrices showing the conditional probability of assessing an equally weighted neuronal population response as being caused by stimulus $i$ when it was actually caused by stimulus $j$ computed from metric space analysis using the van Rossum measure with timescale $\tau=6 \mathrm{~ms}$, and a population size of 27 neurons for chirp evaluation window sizes of $20 \mathrm{~ms}$ (left), $104 \mathrm{~ms}$ (middle), and $1000 \mathrm{~ms}$ (right) with (top) and without (middle) noise correlations. The white dashed lines indicate borders between blocks of stimuli for which a given attribute (e.g., amplitude, duration, phase) was varied. Bottom, Discrimination performance as a function of chirp evaluation window with (black) and without (gray) noise correlation for the unequally weighted case. The dashed line indicates chance level (1 of 36 stimuli). Inset, Performance without noise correlations plotted against performance with noise correlations for the equally weighted case with color-coded chirp evaluation window size. $\boldsymbol{b}$, Top, Same as $\boldsymbol{a}$, but for the unequally weighted case with (top) and without (middle) noise correlations. Bottom, Discrimination performance as a function of chirp evaluation window with (black) and without (gray) noise correlations. The dashed line indicates chance level (1 of 36 stimuli). Inset, Performance without noise correlations plotted against performance with noise correlations for the unequally weighted case with color-coded chirp evaluation window size. 

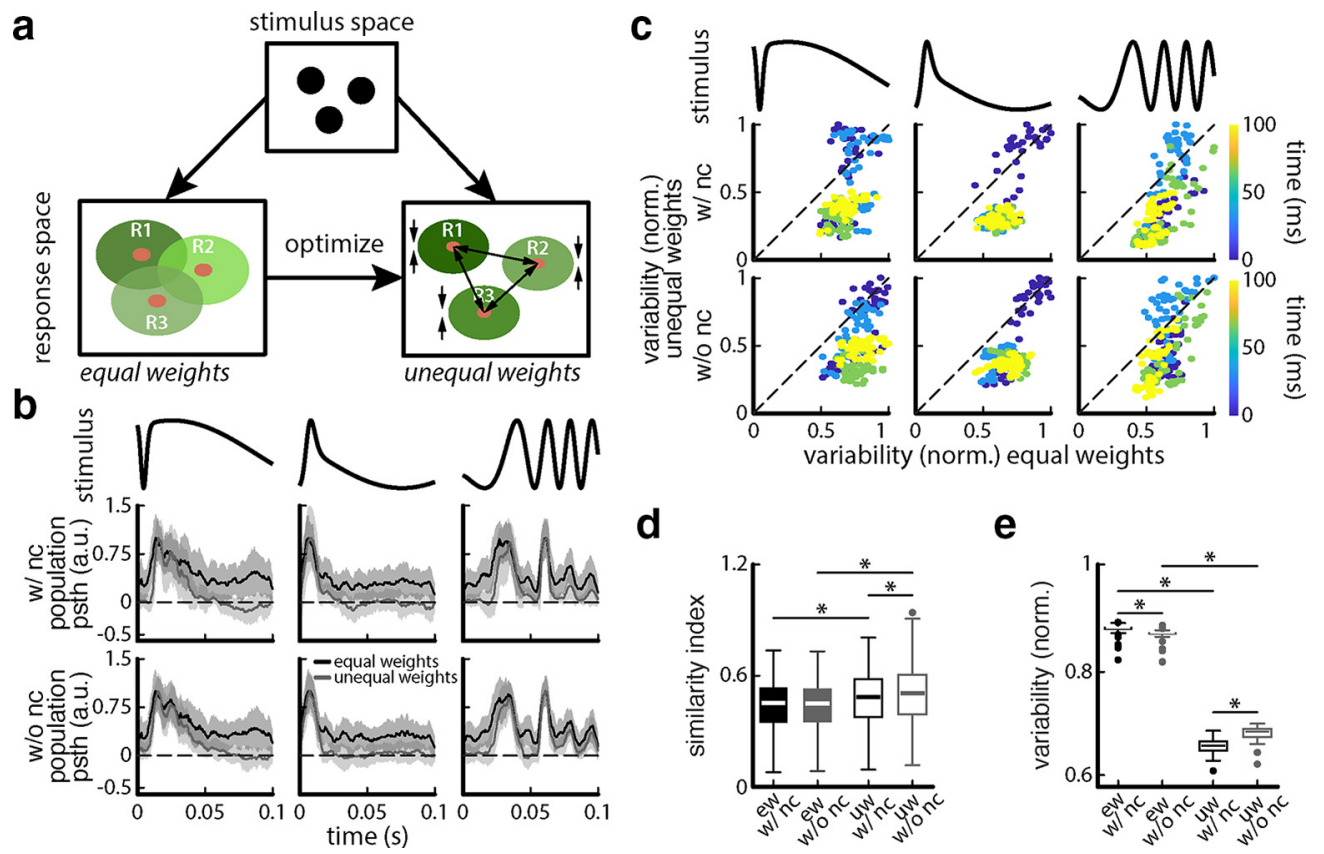

d
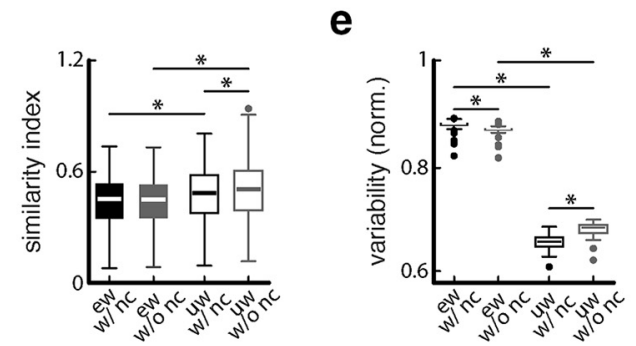

Figure 10. Increased performance is because of increased distance between trial-averaged responses as well as reduction in trial-to-trial variability. $\boldsymbol{a}$, Schematic illustrating the response space of the equally weighted (bottom, left) and unequally weighted (bottom, right) neuronal population to a given stimulus space (top). The EA optimized the response space such as to minimize response variability while increasing their distances. $\boldsymbol{b}$, Population PSTHs to three example chirp stimuli (top) with (middle) and without (bottom) noise correlations. Note that the PSTHs obtained in the unequally weighted (middle, with noise correlations, gray; bottom, without noise correlations, gray) and equally weighted (middle, with noise correlations, black; bottom, without noise correlations, black) cases both tended to follow the stimulus waveform. However, the former displayed greater variation compared with the latter and were thus more discriminable from one another. c, Variability of the unequally weighted population as a function of variability of the equally weighted population with (middle) and without (bottom) noise correlations for three different chirp stimuli (top). Datapoints are color coded according to their time of occurrence after stimulus onset. $\boldsymbol{d}$, Boxplots showing the average SI of the population responses with and without noise correlations for the equally weighted (left) and unequally weighted (right) cases. SI significantly increased in both cases (with noise correlations, $n=630$ : equally weighted (solid black) vs unequally weighted (hollow black); one-way ANOVA: $\mathrm{df}=3 ; F=71.69 ; p=3.19 * 10^{-4}$; without noise correlations, $n=630$ : equally weighted (solid gray) vs unequally weighted (hollow gray); one-way ANOVA: $\mathrm{df}=3 ; F=71.69 ; p=4.55 * 10^{-35}$, Bonferroni corrected). SI was also significantly higher for the unequally weighted population without noise correlations ( $n=630$; one-way ANOVA: $\mathrm{df}=3 ; F=71.69 ; p=3.55 * 10^{-17}$, Bonferroni corrected). $\boldsymbol{e}$, Boxplots showing the normalized average response variability of the population responses with and without noise correlations for the equally weighted (left) and unequally weighted (right) cases within the interquartile range (see Materials and Methods; $n=100$ data points). Response variability across all chirp stimuli significantly decreased for equally weighted sums but actually increased for unequally weighted sums when removing noise correlations (equally weighted: with noise correlations (solid black) vs without noise correlations (solid gray), one-way ANOVA; $\mathrm{df}=3 ; F=1.12 * 10^{4} ; p=1.57 * 10^{-7}$; unequally weighted: with (hollow black) vs without noise correlations (hollow gray, one-way ANOVA; $\mathrm{df}=3 ; F=1.12 * 10^{4} ; p=6.56 * 10^{-38}$, Bonferroni corrected). Note, that the response variability is significantly higher for with and without noise correlations in the equally weighted case (left) compared to the unequally weighted case (right) (with noise correlations: equally (left, solid black) vs unequally weighted (right, hollow black); one-way ANOVA; $\mathrm{df}=3 ; F=1.12 * 10^{4} ; p=0.0001$, Bonferroni corrected; without noise correlations: equally (left, solid gray) vs unequally weighted (right, hollow gray): one-way ANOVA; $\mathrm{df}=3 ; F=1.12 * 10^{4} ; p=1.20 * 10^{-311}$, Bonferroni corrected). $*$ Indicates statistical significance at the $p=0.05$ level using a one-way ANOVA with Bonferroni correction.

corrected), the trial-to-trial variability was significantly higher after shuffling (Fig. 10e: one-way ANOVA: $\mathrm{df}=3, F=1.12 * 10^{4}$, $p=6.56 * 10^{-38}$; Bonferroni corrected). This indicates that the EA identified solutions for each case (i.e., with and without noise correlations), which are different from one another, as a poorer similarity index is compensated for by a more beneficial trial-to-trial variability.

\section{Different weight vectors can optimize performance}

Finally, we investigated variability in the best weight vectors identified by our EA algorithm (i.e., those for which performance is best for a given simulation). For this analysis, we eliminated "trivial" degeneracies in the data, such as those introduced by multiplying a given weight vector by a nonzero constant, which does not alter performance (see Materials and Methods). Figure $11 a$ (left) shows a boxplot of the absolute weights obtained for 20 different simulations for each neuron sorted for performance for each response class with noise correlations. The weights assigned to bcr and br neurons were on average significantly higher than the weights assigned to $\mathrm{nr}$ and $\mathrm{cr}$ neurons [Fig. 11a, right; oneway ANOVA: $\mathrm{df}=3, F=4.98, p_{\mathrm{nr}-\mathrm{br}}=0.02, p_{\mathrm{nr}-\mathrm{bcr}}=0.002, p_{\mathrm{nr}-\mathrm{cr}}$
$=0.72, p_{\mathrm{br}-\mathrm{bcr}}=0.35, p_{\mathrm{br}-\mathrm{cr}}=0.05, p_{\mathrm{bcr}-\mathrm{cr}}=0.005$; least significant difference (LSD) corrected]. Figure $11 b$ (left) shows the same without noise correlations. The weights assigned to bcr and br neurons were on average significantly higher than the weights assigned to $\mathrm{nr}$ and $\mathrm{cr}$ neurons (Fig. $11 \mathrm{~b}$, right; one-way ANOVA: $\mathrm{df}=3, F=3.9, p_{\text {nr-br }}=0.02, p_{\text {nr-bcr }}=0.02, p_{\text {nr-cr }}=0.97, p_{\text {br-bcr }}=$ $0.97, p_{\text {br-cr }}=0.02, p_{\text {bcr-cr }}=0.02$, LSD corrected). Overall, weight vectors displayed clear similarity with one another. Notably, some neurons contributed more to discrimination performance than others (e.g., one br neurons was almost always assigned a weight near unity). We note that this single neuron displayed a discrimination performance of $0.63 \pm 0.01$. Not including this particular neuron and using the evolution algorithm to optimize the weights gave rise to a population performance of $0.55 \pm 0.04$ (with noise correlations) and $0.51 \pm 0.05$ (without noise correlations), respectively. Importantly, this did not affect the qualitative nature of our results, as the discrimination performance of the unequally weighted summed activities was still significantly higher than that of the equally weighted activities (with noise correlations: equal weights, $0.26 \pm 0.01$; unequal weights, $0.55 \pm$ 0.04 ; without noise correlations: equal weights, $0.34 \pm 0.02$; 
unequal weights, $0.51 \pm 0.05$; one-way ANOVA: $\mathrm{df}=3, F=350.71, p_{\mathrm{w} / \mathrm{nc}}=1.49 *$ $10^{-40}, p_{\mathrm{w} / \mathrm{o} \mathrm{nc}}=1.08 * 10^{-26}$, Bonferroni corrected).

However, there were also some notable differences between the obtained weight vectors. For example, one cr neuron was assigned a greater range of weights (Fig. 11a, left). Qualitatively similar results were obtained without noise correlations (Fig. 11b, left). This suggests that there exist multiple weight vectors that give rise to the same level of performance. To test this hypothesis, we took advantage of the fact that similar a performance was obtained with and without noise correlations (Fig. 11c, compare black curves, gray curves). We thus used principal component analysis (see Materials and Methods) to decompose weight vectors into their first three components, which explain most of the variance. It is important to note that we used the same principal components for weight vectors obtained with and without noise correlations. As such, any difference seen has to be because of a difference that existed between both datasets. We then plotted "trajectories" (i.e., how the first three principal components change based on sorting according to increasing performance). We found that these differed from one another (Fig. 11d, inset; one-way ANOVA: $\mathrm{df}=2, F=14.15$, $p_{\mathrm{w} / \mathrm{w} / \mathrm{o}, \mathrm{w} / \mathrm{shuffled}}=1.26 * 10^{-5}, p_{\mathrm{w} / \mathrm{w} / \mathrm{o}, \text { w/o shuf- }}$ fled $=2.74 * 10^{-5}$, Bonferroni corrected), thereby suggesting that different weight vectors can indeed give rise to the same performance. The correlation coefficient between weight vectors increased with increasing performance (Fig. 11e), suggesting that the number of different combinations of weights that give rise to a given performance decreases when higher levels of performance are considered. The existence of nontrivial degeneracies is important as it makes finding one such solution easier, as discussed further below.

\section{Discussion}

Summary of results

We investigated how midbrain electrosensory neuronal populations encoded natural electrocommunication stimuli (i. e., chirps). These chirps consist of highly heterogeneous transient waveforms and furthermore give rise to different phase advances of the underlying background beat. To do so, we recorded the activities of multiple midbrain neurons simultaneously in response to chirps whose attributes were varied systematically. First, in the absence of stimulation, we found that the activities of midbrain neurons were correlated, and that such correlations decayed in magnitude with increasing
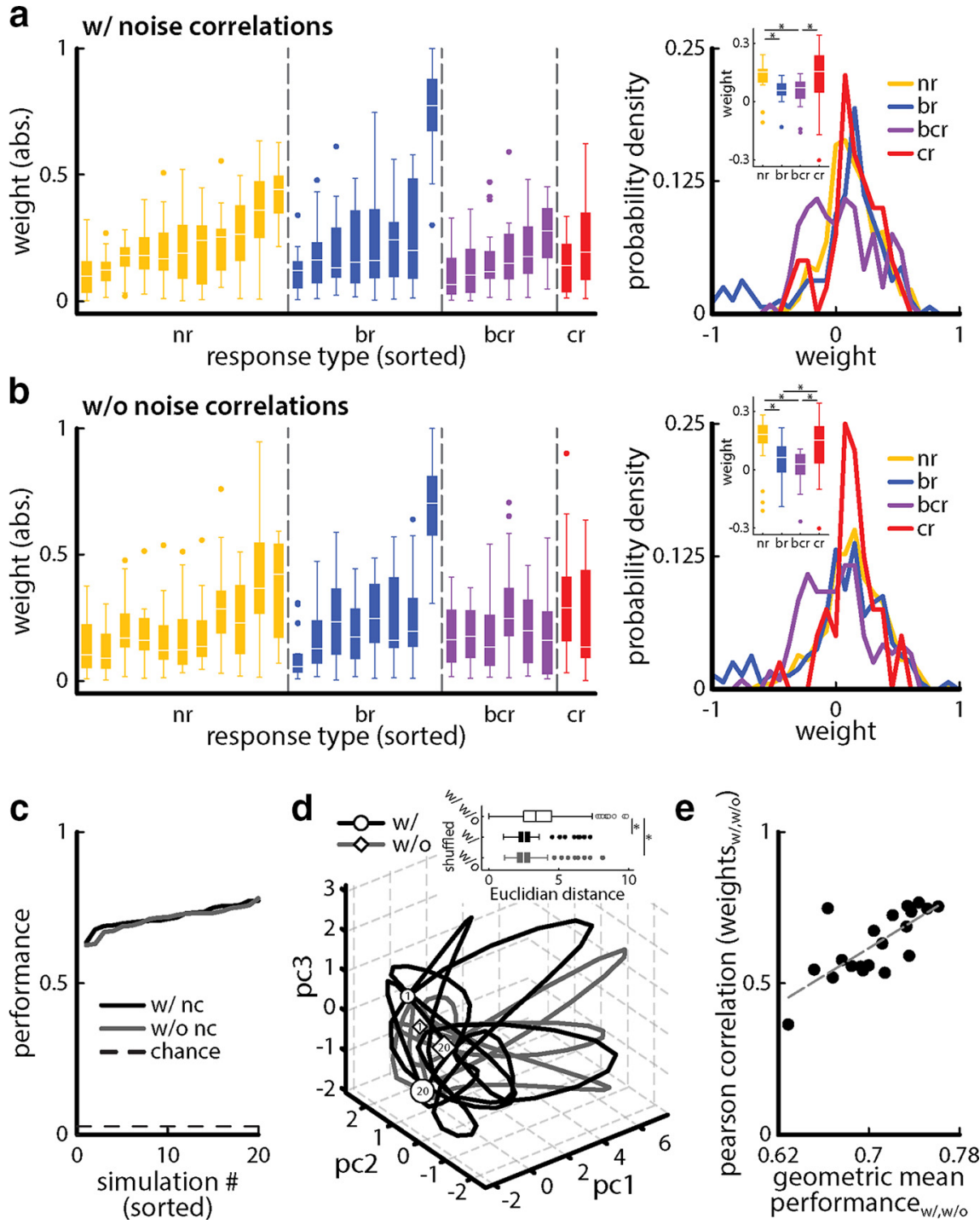

Figure 11. The weight matrices obtained for a neuronal population with (w/) and without (w/0) noise correlations are different. $\boldsymbol{a}$, Left, Boxplot of the normalized absolute weights obtained by the EA and sorted for increasing performance for each response class (yellow, nr; blue, br; purple, bcr; red, cr) over 20 EA realizations (simulations) for a neuronal population ( $n=27)$ with noise correlations. Right, Probability densities of weights obtained for each neuron type. Inset, Boxplots of weights for each neuron type. *Indicates statistical significance at the $p=0.05$ level using a one-way ANOVA with Bonferroni correction. $\boldsymbol{b}$, Same as $\boldsymbol{a}$ but for the same neuronal population $(n=27)$ without noise correlations. $\boldsymbol{c}$, Discrimination performance for the unequally weighted neuronal population obtained after 20 EA simulations with (black) and without (gray) noise correlations sorted in ascending order. Note that both curves overlap. The dashed line indicates chance level ( 1 of 36 stimuli). $\boldsymbol{d}$, Vector space of the first three principal components representing $>60 \%$ of the observed variability computed from the unequally weighted neuronal populations with (black) and without (gray) noise correlations. The size of the circles (with noise correlations) and diamonds (without noise correlations) represent the magnitude of discrimination performance (small symbols, low discrimination values; big symbols, large discrimination values). The numbers within the symbols indicate the first (1) and last (20) sorted simulation number. Inset, The euclidian distance between the $w /$ and $w / 0$ trajectories in $p c$ space is significantly higher than that between the shuffled $w /$ (one-way ANOVA; $d f=2$; $F=14.15 ; p=1.26 * 10^{-5}$ ) and the shuffled w/0 (one-way ANOVA: $\mathrm{df}=2, F=14.15, p=2.74 * 10^{-5}$ ). $\boldsymbol{e}$, Pearson correlation coefficient of sorted weight vectors with and without noise correlations as a function of the geometric mean of the sorted weight vectors with and without noise correlations. Note the positive relationship between the two quantities (Pearson correlation coefficient: $0.74, p=2 * 10^{-4}$ ).

physical distance, suggesting that midbrain neurons will display noise correlations under stimulation. Under stimulation, we found that neural responses, while highly heterogeneous, could be classified into four different groups. The cr neurons tended to respond only to the transient chirp with excitation and responded only weakly or not at all to the background beat, while br neurons tended to respond only to the background beat and 
weakly or not at all to the transient chirp. We found that br neurons responded to the different phase advances of the beat caused by the chirp and could thus discriminate between chirps. The bcr neurons responded to both beat and chirp, while nr neurons did not respond to either the beat or chirp. We next investigated the correlation structure displayed by midbrain neuronal populations in response to chirp stimuli. Overall, we found that signal correlations between midbrain neurons decreased with increasing physical distance for all response classes except bcrbcr and $\mathrm{cr}-\mathrm{cr}$ pairs when considering all possible pairs as well as when only considering pairs with correlation values significantly different from zero. As such, midbrain neurons located closer to one another tended to display more similar responses to a given chirp stimulus than midbrain neurons located farther apart. However, noise correlations were not dependent on physical distance for most response classes except br-br pairs when considering all possible pairs as well as when only considering pairs with correlation values significantly different from zero. Similar results have been found in previous modeling and experimental studies stating that neurons in close vicinity or of the same cluster tend to display larger noise correlations than neurons that are farther apart (for review, see Doiron et al., 2016). This is because closer neurons presumably share more common presynaptic neurons, as suggested by a modeling study (Kriener et al., 2009). Interestingly, both signal and noise correlations did not depend on the particular chirp stimulus presented and were furthermore independent of one another. This lack of dependence on stimulus waveform is unexpected since previous studies have shown that noise correlations generally depend on stimulus attributes (Snyder et al., 2014; Tan et al., 2014; Franke et al., 2016; Zylberberg et al., 2016). We next investigated the performance of a classifier at discriminating between different chirp waveforms using the combined activities of midbrain neurons. When activities were combined using an equally weighted sum, we found that discrimination performance was greatest when combining the activities of bcr neurons, followed by br neurons. Combining the activities of $\mathrm{cr}$ and $\mathrm{nr}$ neurons gave rise to poor performance overall. Noise correlations had a detrimental effect on performance both when varying population size as well as timescale. Next, we used an EA to find whether assigning each neuron a given weight before summation would improve performance. Our results show that considering unequally weighted sums of neuronal activities gave rise to significant improvement in performance, which was achieved by making trial averages to different chirp waveforms more discriminable from one another, as well as reducing trial-to-trial variability, which was achieved in part by strongly attenuating, if not eliminating, noise correlations. Overall, the greatest discrimination performance was achieved by br and bcr neurons, indicating that the algorithm favored different responses because of the different phase advances of the beat caused by chirp stimuli with different attributes, rather than the responses to the actual transient chirp stimulus waveform itself. Indeed, combining the activities of $\mathrm{cr}$ neurons in an equally or unequally weighted manner gave rise to poor discrimination performance that was barely above chance or that was obtained using only nr neurons. Interestingly, we found that there exist nontrivial degeneracies in weight vectors that give rise to a given performance. Specifically, while it is clear that multiplying all weights by the same nonzero constant will not affect discrimination performance, which is a trivial degeneracy. As such, our results show that there are different combinations of weights that are nontrivially related that give rise to similar discrimination performance. We thus conclude that heterogeneous midbrain neuronal populations transmit information to downstream brain areas that enable reliable discrimination of chirp stimulus waveforms if these activities are combined so as to include neurons that respond differentially to the different phase advances of the beat caused by different chirp stimuli, rather than to the chirp stimulus waveform itself. As such, our results have important implications for understanding how downstream brain areas decode information transmitted by heterogeneous neural populations about complex natural stimuli.

\section{Heterogeneities in midbrain neuronal populations and implications for population coding}

Our results are the first to investigate the effects of heterogeneities within TS neurons on population coding of natural electrocommunication stimuli. Specifically, they show that the neurons that respond most selectively to chirp stimuli (i.e., cr neurons) are actually among the least useful at being able to discriminate between different chirps, with performance barely above that of nonresponding neurons. Rather, it is the activities of neurons that respond to the beat and perturbations of the beat (i.e., the phase advance) by the chirp that make the greatest contribution toward discriminability. Similar to cr neurons, nr neurons also did not contribute to the discrimination performance. The existence of such nr neurons is, however, expected given that previous studies have shown that a significant subpopulation of TS neurons respond selectively to a given stimulus category. For example, some TS neurons respond selectively to moving objects, or envelopes, or chirps (Chacron et al., 2009; Chacron and Fortune, 2010; Khosravi-Hashemi et al., 2011; Vonderschen and Chacron, 2011; McGillivray et al., 2012; Sproule et al., 2015). In contrast, other TS neurons respond more broadly to different stimulus categories in a manner that is reminiscent of that displayed by ELL neurons (Vonderschen and Chacron, 2011; Sproule et al., 2015). The identified br and bcr neurons in the current study most likely correspond to "ELL-like" neurons, and our results show that it is these neurons that make the greatest contribution to discrimination performance. Importantly, neurons that respond selectively and nonselectively to sensory input were found throughout all layers of TS and can even coexist within the same layer (Sproule et al., 2015). As such, it is expected that heterogeneous neural responses similar to those reported here will be observed not only when recording across layers but also within a given layer. Further studies are needed to test this prediction.

We note that our results showing that cr neurons are the least useful to discriminate between different chirp waveforms in no way implies that these neurons do not have a function. Rather, because of their invariant responses to chirps, such neurons are most reliable at reporting the occurrence of a chirp rather than its detailed characteristics (i.e., feature detection; Vonderschen and Chacron, 2011; Aumentado-Armstrong et al., 2015; Metzen et al., 2016).

While the fact that we found that a few neurons contributed the most toward improving discrimination performance is similar to what has been seen in other systems when considering sparse coding of natural stimuli (Mizrahi et al., 2014; Yoshida and Ohki, 2020), there are important differences. This is because studies in other systems have shown that the best-performing neurons were most often those that best responded to the stimuli (Mizrahi et al., 2014; Yoshida and Ohki, 2020). In contrast, our results show that the neurons that are most useful toward discrimination are not those that best responded to the chirp stimuli. 


\section{Optimized decoding by weighted summed activities}

Our results have shown that taking an unequally weighted sum of neuronal activities provided much better discriminability of chirp waveforms than an equally weighted sum. Such "combinatorial codes" can recover much more information about the stimulus and are thus advantageous (Seung and Sompolinsky, 1993; Sanger, 1996; Abbott and Dayan, 1999; Reich et al., 2001; Osborne et al., 2008; Liu et al., 2013; Pitkow et al., 2015). Because of this, together with the fact that TS neurons display large heterogeneities in their responses, it is not surprising that an unequally weighted sum performs better than an equally weighted sum. What was more surprising was how improved performance was achieved. Indeed, we found large overlap between the distribution of weights for all cell classes, indicating that the optimized weights for combinatorial code are obtained nontrivially.

On the one hand, combinatorial codes are physiologically realistic as the weights assigned to each neuron could, for example, represent the strength of synaptic connections to a downstream neuron. On the other hand, as one must then keep track of the identity of individual neurons, it is difficult to conceive how the brain would implement them. One possibility is that the weights should be proportional to the reliability of each individual neuron, which then does not require explicit knowledge of neuron identity and does not lead to information loss (Sharpee and Berkowitz, 2019). Our results suggest that another possibility is that, because there exist multiple solutions that give rise to a given performance (i.e., a degeneracy of solutions), it should theoretically be easier to find one such solution. Degeneracy is also expected to provide robustness to combinatorial codes, as seen for homeostasis (Turrigiano, 2011). This has, to our knowledge, not been explicitly demonstrated before.

Another important issue pertains to how a sensory system would actually learn the weights that allow for the best discrimination between different waveforms. It is likely that such learning would occur during development as the animal is exposed to various stimuli during interactions with conspecifics and heterospecifics. Studies performed in songbirds have shown that specific learning rules can optimize information transmission (Jeanne et al., 2013), and we hypothesize that similar learning rules would lead to decoding that maximizes performance. It is likely that learning requires the forebrain, which has recently been the focus of much investigation (Giassi et al., 2012a,b,c; Trinh et al., 2016; Wallach et al., 2018; Fotowat et al., 2019).

Independence of signal and noise correlations in TS neurons Our results show that signal and noise correlations were independent of one another in midbrain electrosensory neurons. It is important to note that the correlation structure observed in TS strongly differs from that seen for ELL neurons for which there is a positive relationship between signal and noise correlations (Hofmann and Chacron, 2018), which resembles that seen in other systems (Averbeck and Lee, 2006; Liu et al., 2013). Since correlation structures in both ELL and TS neurons were obtained for stimuli that had similar contrasts, the observed differences between the correlation structures in TS and ELL are unlikely to be because of the fact that all the stimuli used in this study had similar contrasts. Rather, it should be noted that the correlation structures seen for ELL neurons and in other systems were obtained for artificial stimuli. As such, it is conceivable that the independence of signal and noise correlations seen for midbrain neurons in response to natural electrocommunication stimuli is a signature of optimized coding at the population level based on an adaptation to natural stimulus statistics. In particular, we hypothesize that such a correlation structure enables a combinatorial code for which any detrimental effects of noise correlations can be eliminated by suitably choosing weights without compromising signal quality. Further studies are needed to test these predictions.

We further hypothesize that the independence of noise correlations on recording distance is because of horizontal (i.e., within layer) and vertical (i.e., across layers) connections within TS (Carr et al., 1981; Carr and Maler, 1985) that are activated under stimulation. This is because such long-range connections would have a "normalizing" effect rather than making noise correlations occur because of local connections between neurons. Alternatively, such correlations could also be because of descending projections from the forebrain (Giassi et al., 2012a,b,c). It is well known that noise correlations are strongly dependent on inputs received by neural populations, such as the relative balance between excitation and inhibition (Doiron et al., 2016). Further studies are needed to understand the origins of noise correlations within the TS.

\section{Discrimination of chirp waveforms: implications for behavior}

Our results provide an answer to the ongoing debate as to whether different chirp stimulus waveforms can be discriminated from another by ELL neurons. Indeed, on the one hand, it has been argued that, based on the responses of ELL neurons, chirps could not be discriminated from one another (Marsat et al., 2009; Allen and Marsat, 2018). However, this analysis relied on simple summation of neuronal activities and was furthermore based on nonsimultaneous recordings that were later combined, which ignores the effects of noise correlations. We hypothesize instead that considering an unequally weighted sum of ELL neuron activities will reveal much better discrimination performance. This is because the data-processing inequality states that information can only be retained or lost, but never recovered (Cover and Thomas, 1991); our results imply that the activities of ELL neuronal populations, which provide afferent input to TS neurons, can be used to reliably discriminate between different chirp stimulus waveforms. Further, we hypothesize that ELL pyramidal neurons will display noise correlations under chirp stimulation. This is because noise correlations were seen in response to other stimuli (Chacron and Bastian, 2008) and the fact that ELL pyramidal cells display correlations between their activities in the absence of stimulation (Hofmann and Chacron, 2017).

It is important to note here that, while our results demonstrate that midbrain neuronal populations transmit information that can reliably be used by downstream brain areas to discriminate between different stimulus waveforms, they do not demonstrate that the organism actually takes advantage of this. This is because information is only useful to an organism if it is actually decoded. Previous studies have demonstrated that weakly electric fish display echo responses to chirp stimuli (Hupé and Lewis, 2008; Gama Salgado and Zupanc, 2011). These consist of a fish "responding" to the chirp of the other fish with a chirp of its own, which consists of modulations of its own EOD. Previous studies have shown that weakly electric fish displayed similar echo responses when chirp attributes such as beat phase as well as amplitude and duration were varied (Metzen et al., 2016, 2020). While this suggests that the activities of invariant TS neurons are decoded downstream, these results do not demonstrate that weakly electric fish cannot distinguish between different chirp stimulus waveforms. Importantly, other behavioral assays, 
such as habituation with a given chirp waveform and a test with a different chirp waveform (Allen and Marsat, 2018) or a two-alternative forced-choice task (Graff et al., 2004), show that weakly electric fish can distinguish between different stimuli but have not been used to specifically test whether animals can distinguish between chirp stimulus waveforms with different attributes (e.g., amplitude). Further studies investigating how the activities of TS neurons are decoded in downstream brain areas as well as using different behavioral assays are thus needed to test whether weakly electric fish can indeed discriminate between different chirp waveforms.

\section{Implications for other systems}

Our results provide several new insights that we expect will be applicable to other systems. This is because of known anatomic and physiological similarities between the electrosensory system and sensory modalities in mammals (e.g., vision, audition, vestibular; Clarke et al., 2015). Indeed, as mentioned above, our results show that it is not necessarily the neurons that respond most selectively to a given sensory input that are best at discriminating between different stimulus waveforms. This has important implications for object-background segregation (Olveczky et al., 2003), which must be performed by all modalities to some extent.

While it is now widely agreed that the effects of correlations on information transmission ultimately depend on how neuronal activities are integrated by downstream brain areas (Cohen and Kohn, 2011; Kohn et al., 2016). To this end, studies have used various decoders to predict the effects of correlations. In particular, linear decoders have mostly been used because one can find the best solution analytically (Seung and Sompolinsky, 1993; Zohary et al., 1994; Sanger, 1996; Abbott and Dayan, 1999; Liu et al., 2013; Pitkow et al., 2015). It is, however, important to note that nonlinear decoders can, in theory, extract much more information than linear decoders (Shamir and Sompolinsky, 2004). This is in part because nonlinear decoders can take into account information that is carried by precise spike timing. Temporal codes have been observed across systems and species (Bair and Koch, 1996; Borst and Theunissen, 1999; Johansson and Birznieks, 2004; Uzzell and Chichilnisky, 2004; Butts et al., 2007; Mackevicius et al., 2012; Harvey et al., 2013; Zuo et al., 2015; Jamali et al., 2016, 2019). In particular, our results showing that some TS neurons perform much better at stimulus discrimination than others resemble recent results obtained in the visual cortex where it was shown that a few neurons performed best at image reconstruction (Yoshida and Ohki, 2020). We further note that evolution algorithms have been used extensively in neural networks. Indeed, such algorithms have been used to optimize the architecture of artificial neural networks toward optimizing performance (Angeline et al., 1994; Ding et al., 2013). Our methodology of using an evolution algorithm to find the weights that optimize discrimination performance can thus be used in other systems where it is believed that spike timing carries important information.

\section{References}

Abbott LF, Dayan P (1999) The effect of correlated variability on the accuracy of a population code. Neural Comput 11:91-101.

Allen KM, Marsat G (2018) Task-specific sensory coding strategies are matched to detection and discrimination performance. J Exp Biol 221: jeb170563.

Angeline PJ, Saunders GM, Pollack JB (1994) An evolutionary algorithm that constructs recurrent neural networks. IEEE Trans Neural Netw 5:54-65.
Attias H, Schreiner CE (1997) Low-order temporal statistics of natural sounds. Adv Neural Inf Process Syst 9:27-33.

Aumentado-Armstrong T, Metzen MG, Sproule MK, Chacron MJ (2015) Electrosensory midbrain neurons display feature invariant responses to natural communication stimuli. PLoS Comput Biol 11:e1004430.

Averbeck BB, Lee D (2006) Effects of noise correlations on information encoding and decoding. J Neurophysiol 95:3633-3644.

Averbeck BB, Latham PE, Pouget A (2006) Neural correlations, population coding and computation. Nat Rev Neurosci 7:358-366.

Bair W, Koch C (1996) Temporal precision of spike trains in extrastriate cortex of the behaving macaque monkey. Neural Comput 8:1185-1202.

Bastian J, Schniederjan S, Nguyenkim J (2001) Arginine vasotocin modulates a sexually dimorphic communication behavior in the weakly electric fish Apteronotus leptorhynchus. J Exp Biol 204:1909-1923.

Bastian J, Chacron MJ, Maler L (2002) Receptive field organization determines pyramidal cell stimulus-encoding capability and spatial stimulus selectivity. J Neurosci 22:4577-4590.

Bell C, Maler L (2005) Central neuroanatomy of electrosensory systems in fish. In: Electroreception (Bullock TH, Hopkins CD, Popper AN, Fay RR, eds), pp 68-111. New York: Springer.

Benda J, Longtin A, Maler L (2005) Spike-frequency adaptation separates transient communication signals from background oscillations. J Neurosci 25:2312-2321.

Benda J, Longtin A, Maler L (2006) A synchronization-desynchronization code for natural communication signals. Neuron 52:347-358.

Borst A, Theunissen F (1999) Information theory and neural coding. Nat Neurosci 2:947-957.

Butts DA, Weng C, Jin J, Yeh CI, Lesica NA, Alonso JM, Stanley GB (2007) Temporal precision in the neural code and the timescales of natural vision. Nature 449:92-95.

Carr CE, Maler L (1985) A Golgi study of the cell types of the dorsal torus semicircularis of the electric fish Eigenmannia: functional and morphological diversity in the midbrain. J Comp Neurol 235:207-240.

Carr CE, Maler L (1986) Electroreception in gymnotiform fish: central anatomy and physiology. In: Electroreception. (Bullock TH, Heiligenberg W, eds), pp 319-373. New York: Wiley.

Carr CE, Maler L, Heiligenberg W, Sas E (1981) Laminar organization of the afferent and efferent systems of the torus semicircularis of gymnotiform fish: morphological substrates for parallel processing in the electrosensory system. J Comp Neurol 203:649-670.

Chacron MJ, Bastian J (2008) Population coding by electrosensory neurons. J Neurophysiol 99:1825-1835.

Chacron MJ, Fortune ES (2010) Subthreshold membrane conductances enhance directional selectivity in vertebrate sensory neurons. J Neurophysiol 104:449-462.

Chacron MJ, Doiron B, Maler L, Longtin A, Bastian J (2003) Non-classical receptive field mediates switch in a sensory neuron's frequency tuning. Nature 423:77-81.

Chacron MJ, Toporikova N, Fortune ES (2009) Differences in the time course of short-term depression across receptive fields are correlated with directional selectivity in electrosensory neurons. J Neurophysiol 102:3270-3279.

Clarke SE, Longtin A, Maler L (2015) Contrast coding in the electrosensory system: parallels with visual computation. Nat Rev Neurosci 16:733-744.

Cohen MR, Kohn A (2011) Measuring and interpreting neuronal correlations. Nat Neurosci 14:811-819.

Cover T, Thomas J (1991) Elements of information theory. New York: Wiley.

Ding S, Li H, Su C, Yu J, Fengxiang J (2013) Evolutionary artificial neural networks: a review. Artif Intell Rev 39:251-260.

Doiron B, Litwin-Kumar A, Rosenbaum R, Ocker GK, Josić K (2016) The mechanics of state-dependent neural correlations. Nat Neurosci 19:383393.

Ecker AS, Berens P, Keliris GA, Bethge M, Logothetis NK, Tolias AS (2010) Decorrelated neuronal firing in cortical microcircuits. Science 327:584587.

Engler G, Zupanc GK (2001) Differential production of chirping behavior evoked by electrical stimulation of the weakly electric fish, Apteronotus leptorhynchus. J Comp Physiol A 187:747-756.

Engler G, Fogarty CM, Banks JR, Zupanc GK (2000) Spontaneous modulations of the electric organ discharge in the weakly electric fish, Apteronotus leptorhynchus: a biophysical and behavioral analysis. J Comp Physiol A 186:645-660. 
Fotowat H, Harrison RR, Krahe R (2013) Statistics of the electrosensory input in the freely swimming weakly electric fish Apteronotus leptorhynchus. J Neurosci 33:13758-13772.

Fotowat H, Lee C, Jun JJ, Maler L (2019) Neural activity in a hippocampuslike region of the teleost pallium is associated with active sensing and navigation. Elife 8:e44119.

Franke F, Fiscella M, Sevelev M, Roska B, Hierlemann A, da Silveira RA (2016) Structures of neural correlation and how they favor coding. Neuron 89:409-422.

Gama Salgado JA, Zupanc GKH (2011) Echo response to chirping in the weakly electric brown ghost knifefish (Apteronotus leptorhynchus): role of frequency and amplitude modulations. Can J Zool 89:498-508.

Georgopoulos AP, Schwartz AB, Kettner RE (1986) Neuronal population coding of movement direction. Science 233:1416-1419.

Giassi AC, Harvey-Girard E, Valsamis B, Maler L (2012a) Organization of the gymnotiform fish pallium in relation to learning and memory: I. Cytoarchitectonics and cellular morphology. J Comp Neurol 520:33143337.

Giassi AC, Duarte TT, Ellis W, Maler L (2012b) Organization of the gymnotiform fish pallium in relation to learning and memory: II. Extrinsic connections. J Comp Neurol 520:3338-3368.

Giassi AC, Ellis W, Maler L (2012c) Organization of the gymnotiform fish pallium in relation to learning and memory: III. Intrinsic connections. J Comp Neurol 520:3369-3394

Graff C, Kaminski G, Gresty M, Ohlmann T (2004) Fish perform spatial pattern recognition and abstraction by exclusive use of active electrolocation. Curr Biol 14:818-823.

Harvey MA, Saal HP, Dammann JF 3rd, Bensmaia SJ (2013) Multiplexing stimulus information through rate and temporal codes in primate somatosensory cortex. PLoS Biol 11:e1001558.

Henninger J, Krahe R, Kirschbaum F, Grewe J, Benda J (2018) Statistics of natural communication signals observed in the wild identify important yet neglected stimulus regimes in weakly electric fish. J Neurosci 38:5456-5465.

Hitschfeld EM, Stamper SA, Vonderschen K, Fortune ES, Chacron MJ (2009) Effects of restraint and immobilization on electrosensory behaviors of weakly electric fish. ILAR J 50:361-372.

Hofmann V, Chacron MJ (2017) Differential receptive field organizations give rise to nearly identical neural correlations across three parallel sensory maps in weakly electric fish. PLoS Comput Biol 13:e1005716.

Hofmann V, Chacron MJ (2018) Population coding and correlated variability in electrosensory pathways. Front Integr Neurosci 12:56.

Hupé GJ, Lewis JE (2008) Electrocommunication signals in free swimming brown ghost knifefish, Apteronotus leptorhynchus. J Exp Biol 211:16571667.

Jamali M, Chacron MJ, Cullen KE (2016) Self-motion evokes precise spike timing in the primate vestibular system. Nat Commun 7:13229.

Jamali M, Carriot J, Chacron MJ, Cullen KE (2019) Coding strategies in the otolith system differ for translational head motion vs. static orientation relative to gravity. Elife 8:e45573.

Jeanne JM, Sharpee TO, Gentner TQ (2013) Associative learning enhances population coding by inverting interneuronal correlation patterns. Neuron 78:352-363.

Johansson RS, Birznieks I (2004) First spikes in ensembles of human tactile afferents code complex spatial fingertip events. Nat Neurosci 7:170-177.

Jun JJ, Steinmetz NA, Siegle JH, Denman DJ, Bauza M, Barbarits B, Lee AK, Anastassiou CA, Andrei A, Aydın Ç, Barbic M, Blanche TJ, Bonin V, Couto J, Dutta B, Gratiy SL, Gutnisky DA, Häusser M, Karsh B, Ledochowitsch P, et al. (2017) Fully integrated silicon probes for highdensity recording of neural activity. Nature 551:232-236.

Khosravi-Hashemi N, Fortune ES, Chacron MJ (2011) Coding movement direction by burst firing in electrosensory neurons. J Neurophysiol 106:1954-1968.

Kohn A, Coen-Cagli R, Kanitscheider I, Pouget A (2016) Correlations and Neuronal Population Information. Annu Rev Neurosci 39:237-256.

Kolodziejski JA, Nelson BS, Smith GT (2005) Sex and species differences in neuromodulatory input to a premotor nucleus: a comparative study of substance $\mathrm{P}$ and communication behavior in weakly electric fish. J Neurobiol 62:299-315.

Kriener B, Helias M, Aertsen A, Rotter S (2009) Correlations in spiking neuronal networks with distance dependent connections. J Comput Neurosci 27:177-200.
Lin IC, Okun M, Carandini M, Harris KD (2015) The nature of shared cortical variability. Neuron 87:644-656.

Liu S, Gu Y, DeAngelis GC, Angelaki DE (2013) Choice-related activity and correlated noise in subcortical vestibular neurons. Nat Neurosci 16:8997.

Mackevicius EL, Best MD, Saal HP, Bensmaia SJ (2012) Millisecond precision spike timing shapes tactile perception. J Neurosci 32:15309-15317.

Mante V, Frazor RA, Bonin V, Geisler WS, Carandini M (2005) Independence of luminance and contrast in natural scenes and in the early visual system. Nat Neurosci 8:1690-1697.

Marsat G, Maler L (2010) Neural heterogeneity and efficient population codes for communication signals. J Neurophysiol 104:2543-2555.

Marsat G, Proville RD, Maler L (2009) Transient signals trigger synchronous bursts in an identified population of neurons. J Neurophysiol 102:714723.

McGillivray P, Vonderschen K, Fortune ES, Chacron MJ (2012) Parallel coding of first- and second-order stimulus attributes by midbrain electrosensory neurons. J Neurosci 32:5510-5524.

Mendels OP, Shamir M (2018) Relating the structure of noise correlations in macaque primary visual cortex to decoder performance. Front Comput Neurosci 12:12.

Metzen MG (2019) Encoding and perception of electro-communication signals in Apteronotus leptorhynchus. Front Integr Neurosci 13:39.

Metzen MG, Chacron MJ (2017) Stimulus background influences phase invariant coding by correlated neural activity. Elife 6:e24482.

Metzen MG, Jamali M, Carriot J, Ávila-Åkerberg O, Cullen KE, Chacron MJ (2015) Coding of envelopes by correlated but not single-neuron activity requires neural variability. Proc Natl Acad Sci U S A 112:4791-4796.

Metzen MG, Hofmann V, Chacron MJ (2016) Neural correlations enable invariant coding and perception of natural stimuli in weakly electric fish. Elife 5:e12993.

Metzen MG, Hofmann V, Chacron MJ (2020) Neural synchrony gives rise to amplitude- and duration-invariant encoding consistent with perception of natural communication stimuli. Front Neurosci 14:79.

Meyer JH, Leong M, Keller CH (1987) Hormone-induced and maturational changes in electric organ discharges and electroreceptor tuning in the weakly electric fish Apteronotus. J Comp Physiol A 160:385-394.

Mizrahi A, Shalev A, Nelken I (2014) Single neuron and population coding of natural sounds in auditory cortex. Curr Opin Neurobiol 24:103-110.

Moreno-Bote R, Beck J, Kanitscheider I, Pitkow X, Latham P, Pouget A (2014) Information-limiting correlations. Nat Neurosci 17:1410-1417.

Nelson ME, MacIver MA (1999) Prey capture in the weakly electric fish Apteronotus albifrons: sensory acquisition strategies and electrosensory consequences. J Exp Biol 202:1195-1203.

Olveczky BP, Baccus SA, Meister M (2003) Segregation of object and background motion in the retina. Nature 423:401-408.

Osborne LC, Palmer SE, Lisberger SG, Bialek W (2008) The neural basis for combinatorial coding in a cortical population response. J Neurosci 28:13522-13531.

Pitkow X, Liu S, Angelaki DE, DeAngelis GC, Pouget A (2015) How can single sensory neurons predict behavior? Neuron 87:411-423.

Reich DS, Mechler F, Victor JD (2001) Independent and redundant information in nearby cortical neurons. Science 294:2566-2568.

Sanger TD (1996) Probability density estimation for the interpretation of neural population codes. J Neurophysiol 76:2790-2793.

Schneidman E, Berry MJ 2nd, Segev R, Bialek W (2006) Weak pairwise correlations imply strongly correlated network states in a neural population. Nature 440:1007-1012.

Seung HS, Sompolinsky H (1993) Simple models for reading neuronal population codes. Proc Natl Acad Sci U S A 90:10749-10753.

Shamir M, Sompolinsky H (2004) Nonlinear population codes. Neural Comput 16:1105-1136.

Sharpee TO, Berkowitz JA (2019) Linking neural responses to behavior with information-preserving population vectors. Curr Opin Behav Sci 29:3744.

Simoncelli EP, Olshausen BA (2001) Natural image statistics and neural representation. Annu Rev Neurosci 24:1193-1216.

Snyder AC, Morais MJ, Kohn A, Smith MA (2014) Correlations in V1 are reduced by stimulation outside the receptive field. J Neurosci 34:1122211227. 
Sproule MKJ, Metzen MG, Chacron MJ (2015) Parallel sparse and dense information coding streams in the electrosensory midbrain. Neurosci Lett 607:1-6.

Tan AY, Chen Y, Scholl B, Seidemann E, Priebe NJ (2014) Sensory stimulation shifts visual cortex from synchronous to asynchronous states. Nature 509:226-229.

Toporikova N, Chacron MJ (2009) SK channels gate information processing in vivo by regulating an intrinsic bursting mechanism seen in vitro. J Neurophysiol 102:2273-2287.

Trinh AT, Harvey-Girard E, Teixeira F, Maler L (2016) Cryptic laminar and columnar organization in the dorsolateral pallium of a weakly electric fish. J Comp Neurol 524:408-428.

Turrigiano G (2011) Too many cooks? Intrinsic and synaptic homeostatic mechanisms in cortical circuit refinement. Annu Rev Neurosci 34:89-103.

Uzzell VJ, Chichilnisky EJ (2004) Precision of spike trains in primate retinal ganglion cells. J Neurophysiol 92:780-789.

van Rossum MC (2001) A novel spike distance. Neural Comput 13:751-763.

Vonderschen K, Chacron MJ (2011) Sparse and dense coding of natural stimuli by distinct midbrain neuron subpopulations in weakly electric fish. J Neurophysiol 106:3102-3118.

Wallach A, Harvey-Girard E, Jun JJ, Longtin A, Maler L (2018) A time-stamp mechanism may provide temporal information necessary for egocentric to allocentric spatial transformations. Elife 7:e36769.

Walz H, Hupé GJ, Benda J, Lewis JE (2013) The neuroethology of electrocommunication: how signal background influences sensory encoding and behaviour in Apteronotus leptorhynchus. J Physiol Paris 107:13-25.

Walz H, Grewe J, Benda J (2014) Static frequency tuning accounts for changes in neural synchrony evoked by transient communication signals. J Neurophysiol 112:752-765.
Yoshida T, Ohki K (2020) Natural images are reliably represented by sparse and variable populations of neurons in visual cortex. Nat Commun 11:872.

Zakon H, Oestreich J, Tallarovic S, Triefenbach F (2002) EOD modulations of brown ghost electric fish: JARs, chirps, rises, and dips. J Physiol Paris 96:451-458.

Zohary E, Shadlen MN, Newsome WT (1994) Correlated neuronal discharge rate and its implications for psychophysical performance. Nature 370:140-143.

Zuo Y, Safaai H, Notaro G, Mazzoni A, Panzeri S, Diamond ME (2015) Complementary contributions of spike timing and spike rate to perceptual decisions in rat S1 and S2 cortex. Curr Biol 25:357-363.

Zupanc GK, Sîrbulescu RF, Nichols A, Ilies I (2006) Electric interactions through chirping behavior in the weakly electric fish, Apteronotus leptorhynchus. J Comp Physiol A Neuroethol Sens Neural Behav Physiol 192:159-173.

Zupanc GK, Ilies I, Sîrbulescu RF, Zupanc MM (2014) Large-scale identification of proteins involved in the development of a sexually dimorphic behavior. J Neurophysiol 111:1646-1654.

Zupanc GKH (2002) From oscillators to modulators: behavioral and neural control of modulations of the electric organ discharge in the gymnotiform fish, Apteronotus leptorhynchus. J Physiol (Paris) 96:459-472.

Zupanc GKH, Maler L (1993) Evoked chirping in the weakly electric fish Apteronotus leptorhynchus: a quantitative biophysical analysis. Can J Zool 71:2301-2310.

Zylberberg J, Cafaro J, Turner MH, Shea-Brown E, Rieke F (2016) Directionselective circuits shape noise to ensure a precise population code. Neuron 89:369-383. 